\title{
Ratiometric high-resolution imaging of JC-1 fluorescence reveals the subcellular heterogeneity of astrocytic mitochondria
}

\author{
Vera C. Keil • Frank Funke • Andre Zeug • \\ Detlev Schild • Michael Müller
}

Received: 1 August 2011 /Accepted: 7 August 2011 /Published online: 1 September 2011

(C) The Author(s) 2011. This article is published with open access at Springerlink.com

\begin{abstract}
Using the mitochondrial potential $\left(\Delta \Psi_{\mathrm{m}}\right)$ marker JC-1 (5,5',6,6'-tetrachloro-1, 1',3,3'-tetraethylbenzimidazolylcarbocyanine iodide) and high-resolution imaging, we functionally analyzed mitochondria in cultured rat hippocampal astrocytes. Ratiometric detection of JC-1 fluorescence identified mitochondria with high and low $\Delta \Psi_{\mathrm{m}}$. Mitochondrial density was highest in the perinuclear region, whereas $\Delta \Psi_{\mathrm{m}}$ tended to be higher in peripheral mitochondria. Spontaneous $\Delta \Psi_{\mathrm{m}}$ fluctuations, representing episodes of increased energization, appeared in individual mitochondria or synchronized in mitochondrial clusters. They continued upon withdrawal of extracellular $\mathrm{Ca}^{2+}$, but were antagonized by dantrolene or 2-aminoethoxydiphenylborate (2-APB). Fluo-3 imaging revealed local cytosolic $\mathrm{Ca}^{2+}$ transients with similar kinetics that also were depressed by dantrolene and 2-
\end{abstract}

Authors Vera C. Keil and Frank Funke equally contributed to this work.

V. C. Keil $\cdot$ F. Funke $\cdot$ A. Zeug $\cdot$ D. Schild $\cdot$ M. Müller

DFG Research Center Molecular Physiology of the Brain (CMPB),

Humboldtallee 23,

D-37073 Göttingen, Germany

A. Zeug $\cdot$ D. Schild

Abteilung Neurophysiologie und Zelluläre Biophysik,

Zentrum Physiologie und Pathophysiologie,

Georg-August-Universität Göttingen,

Humboldtallee 23,

D-37073 Göttingen, Germany

V. C. Keil $\cdot$ F. Funke $\cdot$ M. Müller $(\square)$

Abteilung Neuro- und Sinnesphysiologie, Zentrum Physiologie und Pathophysiologie, Georg-August-Universität Göttingen,

Humboldtallee 23,

D-37073 Göttingen, Germany

e-mail: mmuelle7@gwdg.de
APB. Massive cellular $\mathrm{Ca}^{2+}$ load or metabolic impairment abolished $\Delta \Psi_{\mathrm{m}}$ fluctuations, occasionally evoking heterogeneous mitochondrial depolarizations. The detected diversity and $\Delta \Psi_{\mathrm{m}}$ heterogeneity of mitochondria confirms that even in less structurally polarized cells, such as astrocytes, specialized mitochondrial subpopulations coexist. We conclude that $\Delta \Psi_{\mathrm{m}}$ fluctuations are an indication of mitochondrial viability and are triggered by local $\mathrm{Ca}^{2+}$ release from the endoplasmic reticulum. This spatially confined organelle crosstalk contributes to the functional heterogeneity of mitochondria and may serve to adapt the metabolism of glial cells to the activity and metabolic demand of complex neuronal networks. The established ratiometric JC-1 imaging - especially combined with two-photon microscopy - enables quantitative functional analyses of individual mitochondria as well as the comparison of mitochondrial heterogeneity in different preparations and/or treatment conditions.

Keywords Two-photon microscopy · Astrocyte - Organelle interaction · Intracellular calcium stores · Hippocampus .

Calcium microdomain

\section{Introduction}

Mitochondria of various preparations have been reported to differ remarkably in subcellular distribution, membrane potential $\left(\Delta \Psi_{\mathrm{m}}\right)$, and metabolic activity, and they may exist as spherical particles or form clusters and filaments [1, 7, 30, $37,38]$. It is this heterogeneity of mitochondria which appears to define their functional determination and vulnerability to metabolic insults or oxidative damage. Detailed structure/function analyses of mitochondrial heterogeneity 
and identification of the underlying molecular physiological processes will therefore be required to foster the understanding of mitochondrial (dys-)function. For these tasks, sophisticated optical techniques enabling functional real-time analysis of individual mitochondria are essential. Also, a ratiometric approach for the determination of $\Delta \Psi_{\mathrm{m}}$ is desirable, as it would allow a direct comparison of mitochondrial energization among different preparations or experimental conditions independent of the concentration of the $\Delta \Psi_{\mathrm{m}}$ marker applied.

5,5',6,6'-Tetrachloro-1,1',3,3'-tetraethylbenzimidazolylcarbocyanine iodide (JC-1) does allow for such a ratiometric approach $[9,38]$. It accumulates in mitochondria depending on $\Delta \Psi_{\mathrm{m}}$ and is present either as monomer or J-aggregate. The JC-1 monomer predominating in depolarized mitochondria emits green fluorescence $(\sim 530 \mathrm{~nm})$, whereas the oligomer (J-aggregate) forming in mitochondria with potentials more negative than $-140 \mathrm{mV}$ emits red fluorescence $(\sim 590 \mathrm{~nm})[15,17,36,38]$. This formation of J-aggregates is fully reversible [33]. Accordingly, the relative green and red components of JC-1 fluorescence distinguish between mitochondria with high and low $\Delta \Psi_{\mathrm{m}}$ and should also be capable of reporting $\Delta \Psi_{\mathrm{m}}$ changes or fluctuations.

Additional advantages of JC-1 are that it is less sensitive to membrane potential changes of the cell under analysis than the commonly used mitochondrial marker rhodamine 123 (Rh123) and it shows more consistent responses to mitochondrial depolarization [35]. More importantly, JC-1—especially its red fluorescing $\mathrm{J}$-aggregate - responds linearly to $\Delta \Psi_{\mathrm{m}}$ changes $[9,38]$. Used in a ratiometric manner, JC-1 can therefore be expected to report also subtle changes in $\Delta \Psi_{\mathrm{m}}$ more sensitively. In contrast, Rh123, dependent on the concentration used, is prone to self quenching, yields highly non-linear responses, and is a qualitative rather than quantitative dye [16].

However, JC-1 seems to be less well retained within cells than Rh123 [31], but used in a ratiometric approach, such loss of dye during the experiment can be neglected. Another potential drawback of JC-1 might be that the red-fluorescing J-aggregates start forming locally inside mitochondria and hence might not always fill up the entire matrix space $[13,26]$ which may limit its reliability for high-resolution imaging approaches. Nevertheless, JC-1 is being used as a standard indicator in, e.g., flow cytometry $[4,8]$.

To take advantage of the ratiometric properties which are offered by no other $\Delta \Psi_{\mathrm{m}}$ indicator, we used JC-1 and evaluated its feasibility for ratiometric high-resolution imaging approaches on the level of individual mitochondria/mitochondrial structures in cultured rat hippocampal astrocytes. The hippocampus was chosen since it responds promptly to metabolic compromise; astrocytes appeared ideal for the optical recordings, since they were widely spread out, flatly grown, and contained a multitude of clearly distinguishable individual mitochondrial structures. Using high-resolution wide-field and two-photon microscopy, we analyzed the heterogeneity of mitochondrial shape and function. Ratiometric JC-1 imaging reliably unveiled differences in mitochondrial shape, $\Delta \Psi_{\mathrm{m}}$, and apparently, also the sensitivity to metabolic challenge of individual mitochondria in their cellular environment. Furthermore, JC-1 imaging revealed spontaneous $\Delta \Psi_{\mathrm{m}}$ fluctuations which were proven by subcellular activity correlation imaging to be synchronized within parts of the mitochondrial population. Mechanistically, these $\Delta \Psi_{\mathrm{m}}$ fluctuations were triggered by localized $\mathrm{Ca}^{2+}$ release from the endoplasmic reticulum (ER). Accordingly, mitochondria-ER crosstalk seems capable of initiating fluctuations in mitochondrial energization, which may serve to metabolically adjust glial cells to the activity of neighboring neurons.

\section{Materials and methods}

\section{Cell cultures}

Cell cultures of hippocampal neurons and glial cells were prepared from 2 to 4-day-old Sprague-Dawley rats as described earlier [19, 21]. After decapitation, the brain was removed and placed in ice-cold Hanks'-balanced salt solution containing 20\% fetal calf serum (FCS; Biochrom). The hippocampi were isolated, cut into small pieces, and trypsinated $\left(5 \mathrm{mg} / \mathrm{ml}, 10 \mathrm{~min}, 37^{\circ} \mathrm{C}\right)$. Cells were then dissociated and centrifuged $\left(1,500 \mathrm{rpm}, 10 \mathrm{~min}, 4^{\circ} \mathrm{C}\right)$. The pellet was re-suspended and plated on Matrigel (BD Biosciences) -coated glass cover slips, which were transferred to 4-well culture plates (Nunc). Cultures were incubated at $37^{\circ} \mathrm{C}$ in a humidified, $5 \% \mathrm{CO}_{2}$-containing atmosphere. After $24 \mathrm{~h}$, the medium was exchanged with growth medium, and after 3 days, medium and growth factors were refreshed. Within 2-3 days in culture, cells fully recovered well-pronounced cellular processes and were suitable for the experiments. Astrocytes were identified by their characteristic flatly grown shape and markedly larger size than neurons.

\section{Solutions}

Chemicals, unless otherwise mentioned, were obtained from Sigma-Aldrich. The artificial cerebrospinal fluid (ACSF) was composed of (in millimolar): $130 \mathrm{NaCl}, 3.5$ $\mathrm{KCl}, 1.25 \mathrm{NaH}_{2} \mathrm{PO}_{4}, 24 \mathrm{NaHCO}_{3}, 1.2 \mathrm{CaCl}_{2}, 1.2 \mathrm{MgSO}_{4}$, and 10 dextrose; aerated with $95 \% \mathrm{O}_{2}-5 \% \mathrm{CO}_{2}$ to adjust $\mathrm{pH}$ to 7.4. Minimum essential cell culture medium (MEM, Invitrogen) was supplemented with $5 \mathrm{mg} / \mathrm{ml}$ glucose, $0.2 \mathrm{mg} / \mathrm{ml} \mathrm{NaHCO}$, and $0.1 \mathrm{mg} / \mathrm{ml}$ transferrin (Calbiochem/Merck). For initial plating, it also contained $10 \%$ 
FCS, $2 \mathrm{mM}$ L-glutamine, and $25 \mu \mathrm{g} / \mathrm{ml}$ insulin. The medium used after day 1 in culture ("growth medium") contained 5\% FCS, $0.5 \mathrm{mM}$ L-glutamine, $20 \mu \mathrm{l} / \mathrm{ml} \mathrm{B} 27$ $50 \times$ supplement (Invitrogen), and $100 \mu \mathrm{g} / \mathrm{ml}$ penicillinstreptomycin (Biochrom).

JC-1 (Invitrogen) and Rh123 were dissolved in dimethylsulfoxide (DMSO) as 2 and $20 \mathrm{mg} / \mathrm{ml}$ stocks, respectively. MitoTracker Red and ER-Tracker Blue-White DPX (both Invitrogen) were dissolved as $1 \mathrm{mM}$ stocks in DMSO. Fluo-3 AM (Mobitec) was prepared as 2-mM stock in DMSO, adding $10 \%$ pluronic acid to improve cell loading. 4',6-Diamidino-2-phenylindol (DAPI), carbonyl cyanide 4(trifluoromethoxy) phenylhydrazone (FCCP; Tocris), and dantrolene were dissolved as 10-20 mM stocks in DMSO; 2-aminoethoxydiphenylborate (2-APB) was dissolved as $100-\mathrm{mM}$ stock in DMSO. Cyanide, azide, and glutamate (all obtained as sodium salts) were prepared as aqueous 1$\mathrm{M}$ stocks. DMSO concentrations of the final solutions were $\leq 0.2 \%$.

\section{Optical recordings}

Functional time-lapse recordings of mitochondria were mostly performed with a fluorescence imaging system composed of a Xenon light source (Polychrome II, Till Photonics) and a sensitive CCD camera (Imago QE, 62\% quantum efficiency at $500 \mathrm{~nm}$, PCO Imaging) attached to an upright microscope (Axiotech Vario, Zeiss). JC-1 was excited at $490 \mathrm{~nm}$ and an optical image splitter device (Dual-View $^{\mathrm{TM}}$, Optical Insights) was attached to the microscope to separate spectrally the green and red components of JC-1 fluorescence (see Fig. 2d for schematic and filter settings). For fluo 3-AM recordings a $505 \mathrm{~nm}$ beamsplitter, a 535/35 $\mathrm{nm}$ bandpass filter (emitter) and 485-nm excitation wavelength were used. Experiments were performed in a submersion style chamber; cell cultures were continuously superfused with $\mathrm{ACSF}\left(32-33^{\circ} \mathrm{C}\right.$, flow rate $3-4 \mathrm{ml} / \mathrm{min}$ ) and $63 \times$ or $100 \times$ water immersion objectives (Apochromat and Achroplan, respectively; Zeiss) were used.

High-resolution ratiometric JC-1 analyses were performed using a custom-built two-photon laser scanning microscope (TPLSM) [29]. The original system layout has been extended by a second upright microscope (BX51WI, Olympus) equipped with an IR-optimized $20 \times 0.95 \mathrm{NA}$ objective (XLUMPlanFL, Olympus) and two nondescanned single-photon counting photomultiplier tubes (H7421-40, Hamamatsu; Fig. 2a). Also a new laser (Mai Tai eHP DS, Newport Spectra-Physics) has been added just recently. The general scan head design was unchanged, but improved and faster galvanometric scanners (VM500C with MiniSAX control circuits and $4 \mathrm{~mm}$ mirrors; General Scanning/Cambridge Technology) were chosen. The scanning process and data acquisition were controlled by a digital signal processor (ADwin-Gold-ENET; Jäger) and the outputs of the two photomultiplier tubes (TTL pulses) were analyzed by a custom-built 2-channel TTL pulse counter. $Z$ axis (axial) adjustment was realized by a piezo-driven objective nano-positioning system (Pifoc P-721; Physik Instrumente). For two-photon imaging, the excitation wavelength was 790-800 nm; fluorescence was separated using a 670-nm dichroic mirror (670dcxxr) and the green and red components were further separated by a $570-\mathrm{nm}$ dichroic mirror (570dcxr) followed by 536/40 and 617/ $73 \mathrm{~nm}$ bandpass emission filters, respectively. Offline image analysis was performed with Tillvision 4.0 (Till Photonics) and MetaMorph Offline 6.1/7.0 (Molecular Devices).

\section{Statistics}

Data were obtained from 35 rat pups (32 different litters) and each experimental paradigm was performed on at least three different animals. Data are given as mean \pm standard deviation.

\section{Results}

Taking advantage of the ratiometric properties of JC-1, we analyzed the subcellular heterogeneity of mitochondria in rat hippocampal astrocytes. The focus was on their cellular distribution, morphology, static/dynamic differences in $\Delta \Psi_{\mathrm{m}}$, and their responses to metabolic challenge.

\section{Heterogeneity in mitochondrial shape and distribution}

Fluorescent labeling of mitochondria with either JC-1 (1 $\mu \mathrm{g} / \mathrm{ml}, 15 \mathrm{~min})$, Rh123 (2.5 $\mu \mathrm{g} / \mathrm{ml}, 15-20 \mathrm{~min})$, or MitoTracker Red (1 $\mu \mathrm{g} / \mathrm{ml}, 10 \mathrm{~min})$ revealed that mitochondria were distributed irregularly, resulting in regions of high and low mitochondrial content within astrocytes (Fig. 1a). As previously reported for various other cell types [1, 7, 30, 37, 38], the morphology of mitochondria was also highly variable in hippocampal astrocytes; mitochondrial shapes ranged from granular to long filamentous structures and their lengths were between 0.8 and $24 \mu \mathrm{m}$. Morphometric analysis of 1,373 mitochondria from 23 JC-1-labeled astrocytes yielded an average mitochondrial length of $3.1 \pm 2.1 \mu \mathrm{m}$. MitoTracker Red labeling revealed a comparable mitochondrial length of $2.2 \pm 1.2 \mu \mathrm{m}$ $(n=506)$. The majority of JC-1-labeled mitochondria $(86 \%)$, measured between 1 and $5 \mu \mathrm{m}$ in length, $1.2 \%$ of mitochondria appeared granular $(\leq 1.0 \mu \mathrm{m}$ length $)$ and $12.8 \%$ were longer than $5 \mu \mathrm{m}$ (Fig. 1b). Particularly long mitochondria were usually found in the less densely packed periphery of the cell.

In the majority of astrocytes (77\% of 114 analyzed) the mitochondrial density was highest in the perinuclear region 

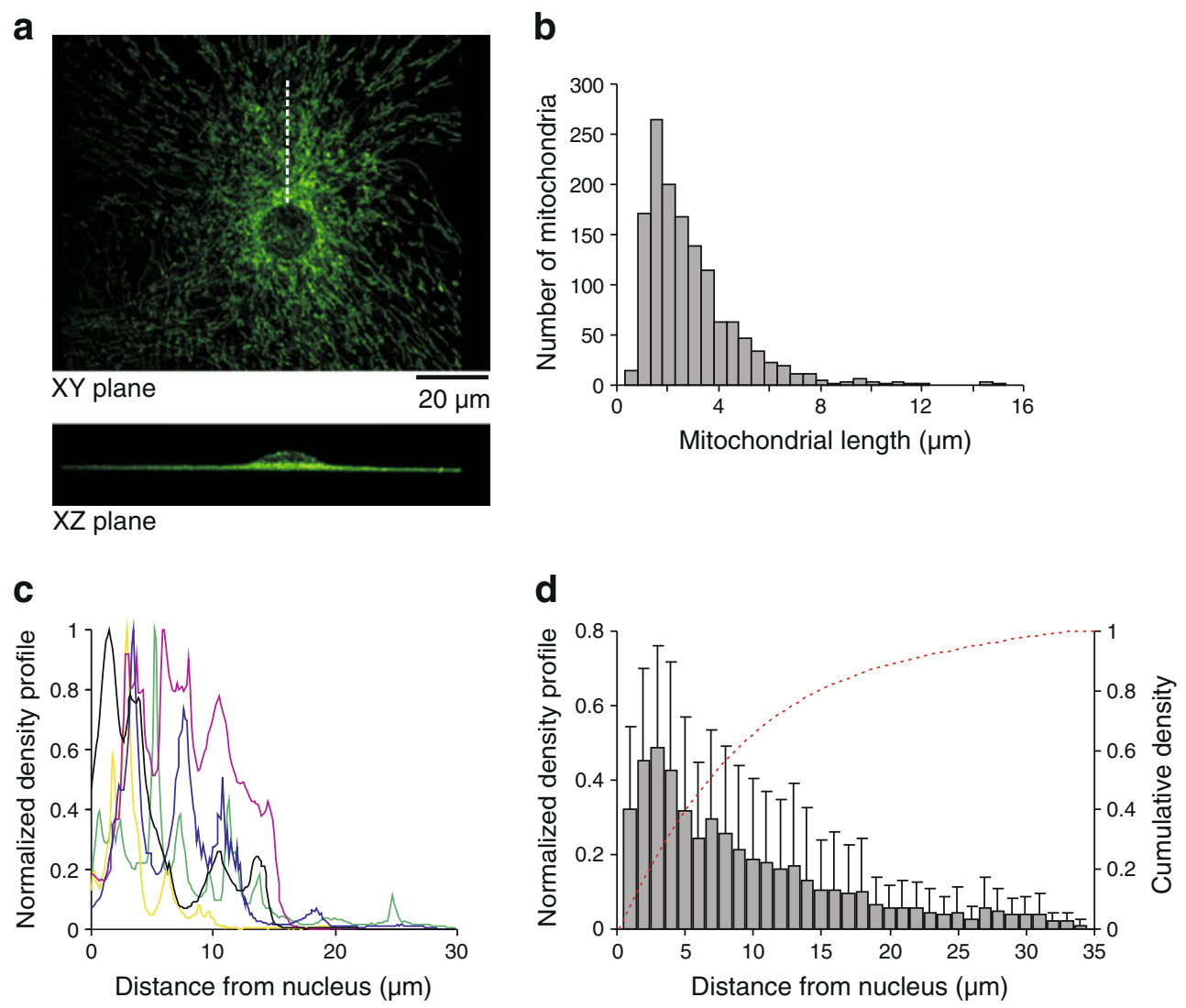

Fig. 1 Subcellular heterogeneity of mitochondrial distribution and shape. a Distribution of Rh123-labeled mitochondria in a cultured hippocampal astrocyte. Note the variable shape of the individual mitochondrial structures and their dense packing around the nucleus. Mitochondria were visualized with a custom-built two-photon laser scanning microscope (TPLSM) using a $63 \times$ objective and $800-\mathrm{nm}$ excitation wavelength; the dashed line resembles a typical line of interest along which the density distribution of mitochondria was calculated. Shown is a 3-dimensional reconstruction composed of 45 optical planes (z-distance $0.25 \mu \mathrm{m}, 250-\mathrm{nm}$ pixel resolution). b Frequency distribution of mitochondrial lengths (bin width $0.5 \mu \mathrm{m}$ ), as analyzed from 1,373 individual JC-1-labeled mitochondria in 23

randomly chosen astrocytes. c Mitochondrial density profiles showing that the mitochondrial packing is highest within a $15-\mu \mathrm{m}$ perimeter around the outer edge of the nucleus and then drops off towards the cellular periphery. The five displayed profiles were calculated along lines of interest, spanning from the edge of the nucleus (distance 0 ) to the periphery (see dashed line in a) and normalized to the most intense density detected in each profile. d Summary of mitochondrial density. Plotted is the normalized averaged mitochondrial density profile of 39 randomly chosen cells (mean \pm standard deviation). The cumulative density curve (dashed curve) shows that $50 \%$ of mitochondria are within $8 \mu \mathrm{m}$ of the nucleus

and dropped off towards the cellular periphery. Calculating a normalized density distribution of mitochondria along a cell-spanning horizontal or vertical line (see dotted line in Fig. 1a) in 39 astrocytes confirmed that on average $50 \%$ of mitochondria were closer than $8 \mu \mathrm{m}$ to the edge of the nucleus (Fig. 1c, d).

Taking advantage of the capability of our TPLSM for dual wavelength detection (Fig. 2a), we counter-labeled mitochondria (MitoTracker Red) and either the nucleus or the ER. Staining with the nucleic acid marker DAPI (7 $\mu \mathrm{M}, 20 \mathrm{~min}$ ) confirmed the high mitochondrial density close to the nucleus (Fig. 2b). Labeling of the ER with ER-Tracker Blue-White DPX (1 $\mu \mathrm{M}, 20 \mathrm{~min})$ suggests that especially perinuclear mitochondria are colocalized with the ER (Fig. 2c).
Heterogeneity of $\Delta \Psi_{\mathrm{m}}$

To analyze $\Delta \Psi_{\mathrm{m}}$ in detail, green and red JC-1 fluorescence were monitored simultaneously using either the dual-detector TPLSM (Fig. 2a) or CCD camera imaging and an optical image splitter device (Dual-View; Fig. 2d, e). The TPLSM was superior in terms of contrast, apparent resolution, and alignment of the "red" and "green" images. The Dual-View/ CCD camera approach offers higher frame rates and due to less intense illumination also enables longer time frame recordings. Thus, depending on the experimental protocol, either the TPLSM (frame rate $0.1 \mathrm{~Hz}$ ) or Dual-View/CCD camera imaging (frame rate $0.2 \mathrm{~Hz}$ ) was used.

Two-photon excitation of JC-1-labeled cells allowed for the real-time analysis of $\Delta \Psi_{\mathrm{m}}$ in individual mitochondria. 
Fig. 2 Dual emission imaging of mitochondrial arrangement and $\Delta \Psi_{\mathrm{m}}$. a General system layout of the TPLSM. Fluorescence emission is spectrally separated onto the two singlephoton counting photomultiplier tubes (PMTs) by a dichroic (e.g., $570 \mathrm{~nm}$ beamsplitter to separate green and red JC-1 fluorescence) and appropriate emission bandpass filters. b The visualization of MitoTracker Redlabeled mitochondria and the DAPI-labeled nucleus (shown in blue) confirms the perinuclear clustering of mitochondria. c Colocalization of MitoTracker Red-labeled mitochondria (shown in red) and the ERTracker Blue-White DPXlabeled ER (shown in green). The overlap of red and green structures suggests that especially perinuclear mitochondria are in close contact with the ER. d General principle of the image splitter device (Dual-View) used for longer time frame ratiometric imaging. The Dual-View is equipped with the appropriate dichroic and bandpass emitters to spectrally separate green and red JC-1 fluorescence and to project the resulting half-images side by side onto the CCD camera chip. e Comparing the green and red half-images enables colocalization and ratiometric studies to analyze mitochondrial heterogeneity and $\Delta \Psi_{\mathrm{m}}$ fluctuations within a cell a

NIR-Excitation $(800-870 \mathrm{~nm})$

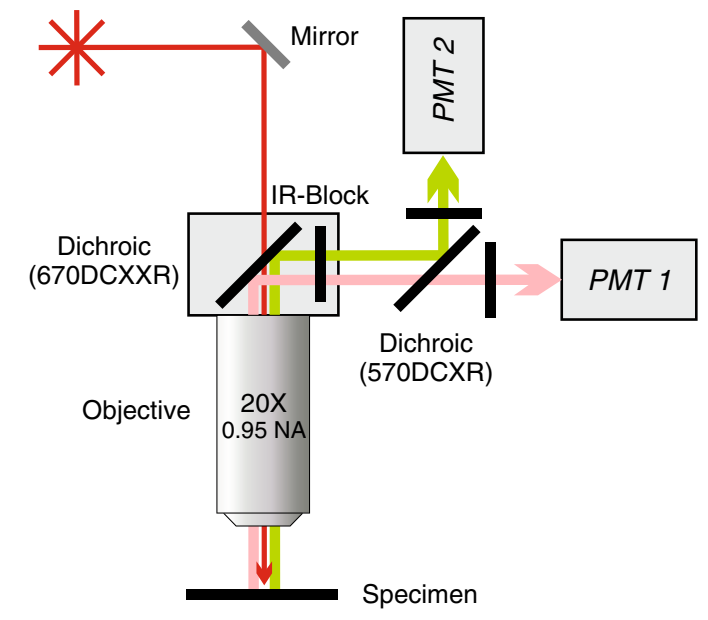

d

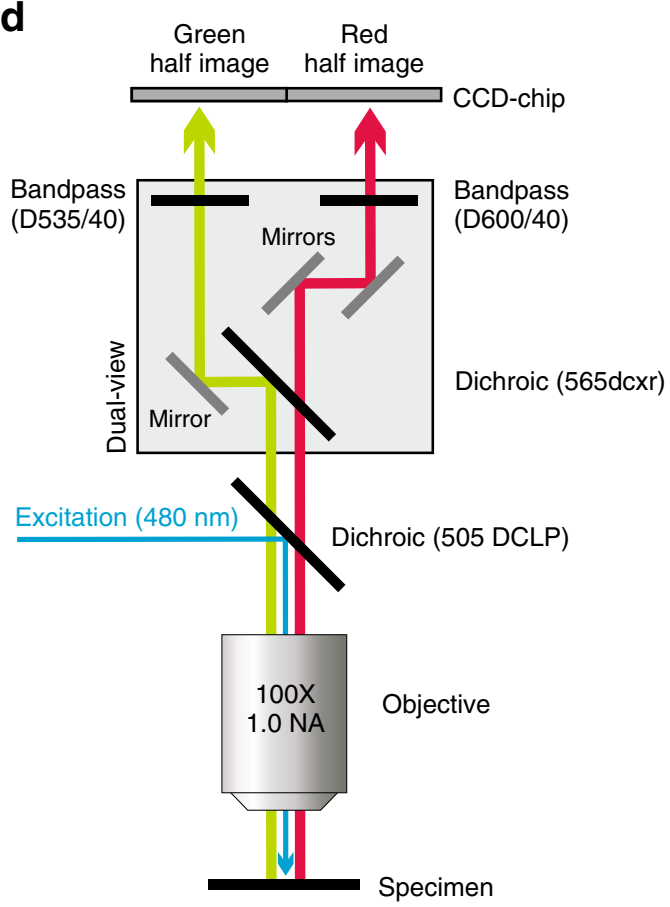

b

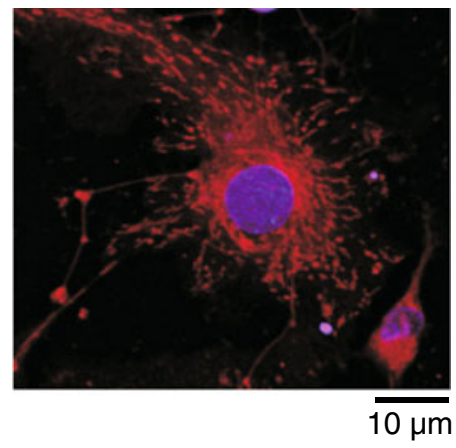

C

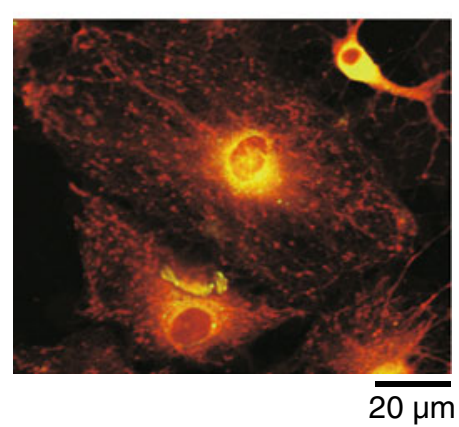

e
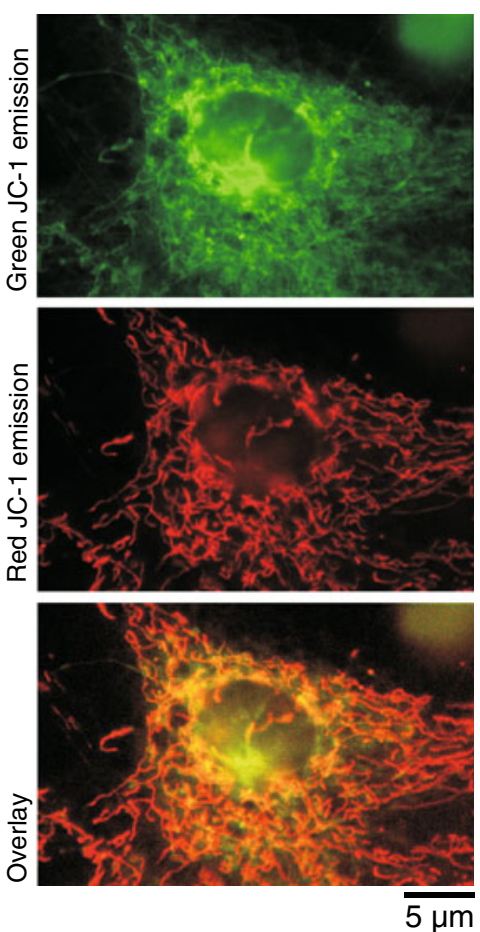

Due to perfect alignment of the two images, we established an online ratiometric analysis of JC-1 fluorescence (ratio= $\mathrm{JC}-1_{\text {red }} * 5 / \mathrm{JC}-1_{\text {green }}$; the factor " 5 " transformed the ratiometric results into an 8-bit scale of 256 levels). The resulting ratiometric image was expressed in pseudo colors, yielding a map of the cell which reports the $\Delta \Psi_{\mathrm{m}}$ of each individual mitochondrion; mitochondria with high $\Delta \Psi_{\mathrm{m}}$ are displayed in warm (red/yellow) colors, whereas depolarized mitochon- dria are displayed in cold (blue/green) colors (Fig. 3a-c). Thus, the functional heterogeneity of mitochondria can be evaluated instantly, which facilitated the selection of proper specimens for the experiments and enabled region-ofinterest-based online analyses during the recordings. The JC-1 ratios somewhat differed, being larger for TPLSM than for Dual-View/CCD camera imaging, since two-photon excitation less efficiently excites the JC-1 monomer [11]. 
Fig. 3 Heterogeneity of $\Delta \Psi_{\mathrm{m}}$ within the mitochondrial population. a-c TPLSM images of JC-1-labeled mitochondria. Displayed are the calculated ratios of red and green JC-1 fluorescence $\left(\right.$ ratio $\left.=F_{\text {red }} * 5 / F_{\text {green }}\right)$.

With the superior contrast and apparent resolution of twophoton imaging, the ratiometric approach allows for a quantitative analysis of $\Delta \Psi_{\mathrm{m}}$ on the level of individual mitochondrial structures (granules, filaments). Note that $\Delta \Psi_{\mathrm{m}}$ clearly differs within the mitochondrial population. Scaling is identical for all three images. d Analyzing the $\Delta \Psi_{\mathrm{m}}$ of individual mitochondria (quantified as JC-1 ratio) shows a bell-shaped distribution with various grades in between functional and depolarized. Data are based on 1,373 individual mitochondria in 23 randomly chosen astrocytes. e A correlation of mitochondrial length and $\Delta \Psi_{\mathrm{m}}$ (JC-1 ratio) could not be proven ( $n=1,373$ mitochondria; 23 astrocytes). $\mathbf{f}$ A correlation of the distance of a given mitochondrion from the nucleus and its $\Delta \Psi_{\mathrm{m}}$ does not exist either. Note, however, that the variability of $\Delta \Psi_{\mathrm{m}}$ seems less pronounced for those mitochondria located within $15 \mu \mathrm{m}$ of the center of the nucleus a

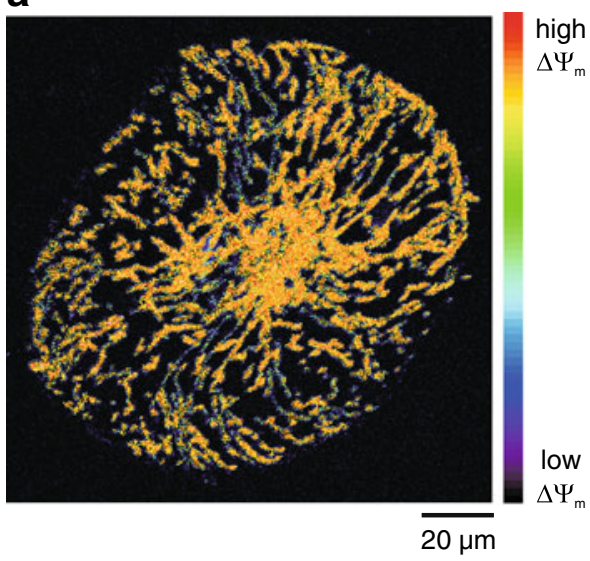

C

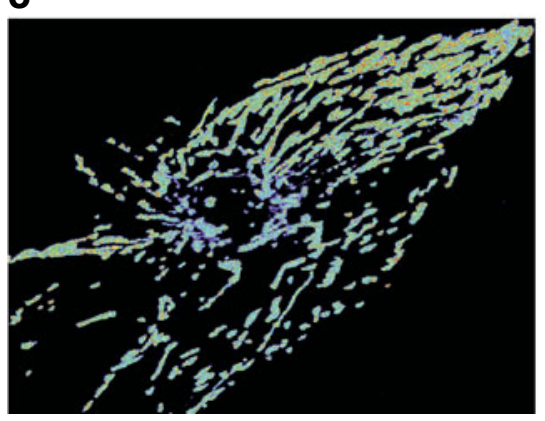

e

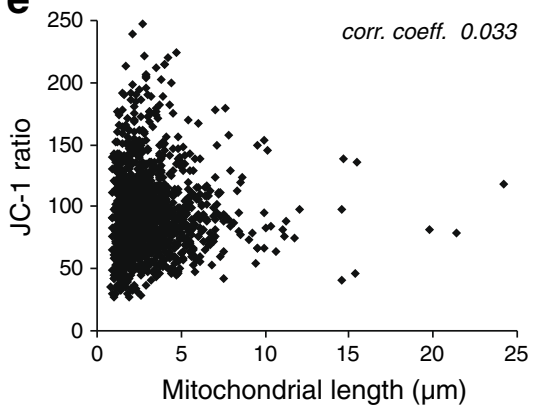

b

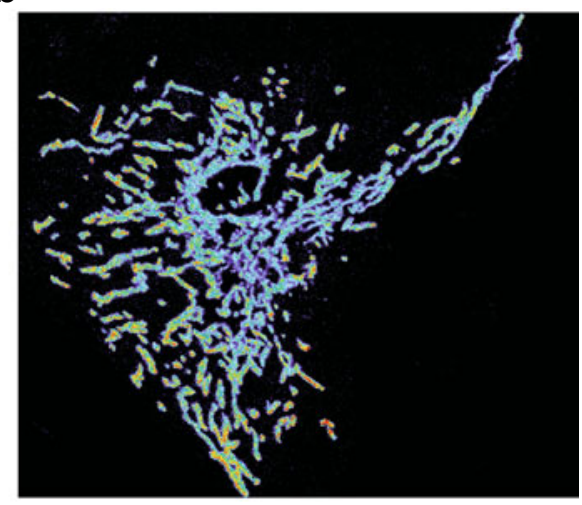

d

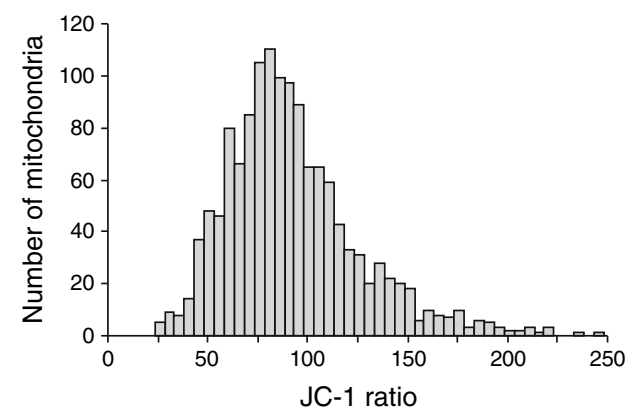

f

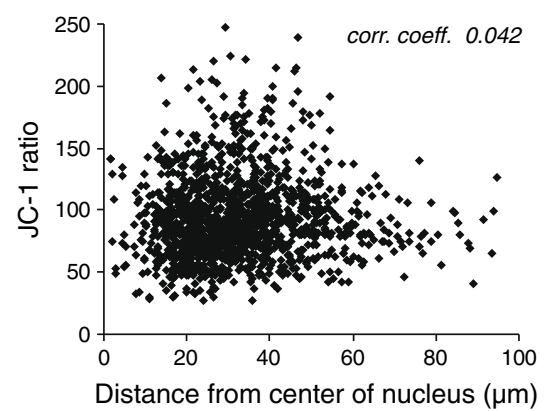

Detailed ratiometric analysis revealed that $\Delta \Psi_{\mathrm{m}}$ was just as heterogeneous as mitochondrial shapes, and that mitochondria with clearly differing potentials coexist even in close proximity within a given cell. Analysis of 1,373 individual mitochondria in 23 randomly chosen astrocytes showed that $\Delta \Psi_{\mathrm{m}}$ ranged in between a JC-1 ratio of 28 (depolarized) and 247 (polarized) and that its scatter yields a bell-shaped, continuous distribution rather than two distinct classes of functional (polarized) and resting (depolarized) mitochondria (Fig. 3d). Accordingly, the majority of mitochondria show an intermediate $\Delta \Psi_{\mathrm{m}}$. A correlation of mitochondrial length and $\Delta \Psi_{\mathrm{m}}$ does not exist $(n=1,373$ mitochondria, 23 astrocytes, correlation coefficient 0.033 ; Fig. 3e).

Testing for a putative effect of the cellular location on $\Delta \Psi_{\mathrm{m}}$ revealed that in 14 out of 23 analyzed astrocytes peripherally located mitochondria exhibited more negative potentials, five cells did not show any noticeable regional differences in $\Delta \Psi_{\mathrm{m}}$, and four cells contained mitochondria with more intense potentials in the perinuclear region. Pooling all 1,373 analyzed mitochondria and testing for a dependence of $\Delta \Psi_{\mathrm{m}}$ and nuclear distance failed, however, to confirm a general correlation (correlation coefficient 0.042; Fig. 3f). However, the reduced scatter of $\Delta \Psi_{\mathrm{m}}$ in perinuclear mitochondria located within $15 \mu \mathrm{m}$ of the center of the nucleus suggests somewhat more uniform potentials of more centrally located mitochondria.

Spontaneous $\Delta \Psi_{\mathrm{m}}$ fluctuations

As reported for mitochondria in various neuronal preparations earlier [3, 30, 39] $\Delta \Psi_{\mathrm{m}}$ was not necessarily constant 
Fig. 4 Spontaneous $\Delta \Psi_{\mathrm{m}}$ fluctuations in mitochondrial clusters and individual mitochondria. a Time-lapse recording (CCD camera/Dual-View, frame rate is $0.2 \mathrm{~Hz}$ ) of synchronized $\Delta \Psi_{\mathrm{m}}$ fluctuations in a cluster of densely packed mitochondria. Note the opposite changes in red and green $\mathrm{JC}-1$ fluorescence. Black arrows identify episodes of increased mitochondrial potential. The displayed images show the perinuclear mitochondrial cluster that was analyzed. b Plotted is the red JC-1 fluorescence of three individual mitochondria (arrow marks) which undergo spontaneous $\Delta \Psi_{\mathrm{m}}$ fluctuations. The rise in red JC-1 fluorescence indicates periods of increased mitochondrial energization. Even though spatially separated mitochondria were analyzed, their $\Delta \Psi_{\mathrm{m}}$ fluctuations are synchronized (correlation coefficients Mito1/2 $=0.58$, Mito1/3=0.93, Mito 2/3 $=0.81$ ). Time scaling is identical for b-d. c Desynchronized $\Delta \Psi_{\mathrm{m}}$ fluctuations of another three individual JC-1-labeled mitochondria. d $\Delta \Psi_{\mathrm{m}}$ fluctuations also occurred in Rh123-labeled mitochondria, yet the frequency was higher than with $\mathrm{JC}-1$.

Note also that Rh123 was more prone to photobleaching
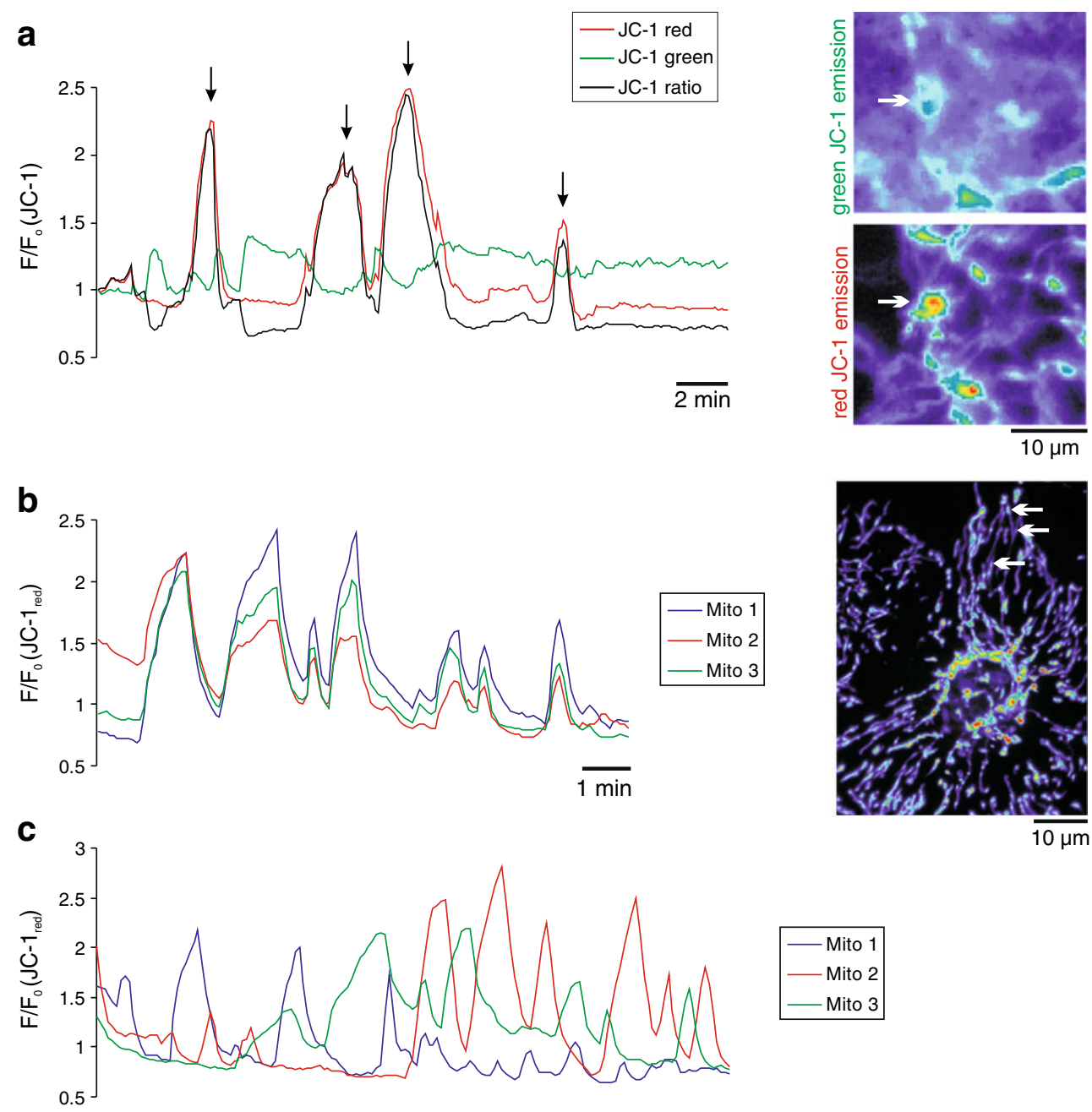

$10 \mu \mathrm{m}$

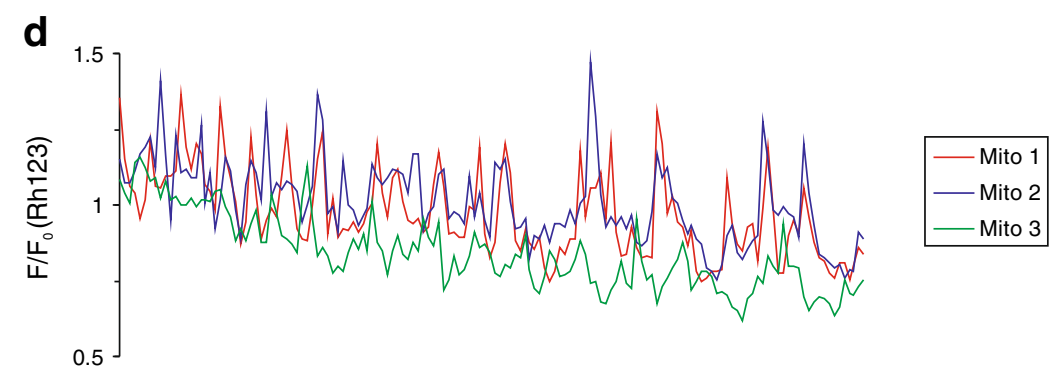

over time. In cultured astrocytes, occasional fluctuations of $\Delta \Psi_{\mathrm{m}}$ occurred and they were most obvious in the red JC-1 fluorescence channel of the CCD camera/Dual-View recordings. Red JC-1 fluorescence clearly increased up to three-fold, whereas green JC-1 fluorescence showed less intense and opposite changes (see Fig. 4a). Accordingly, these $\Delta \Psi_{\mathrm{m}}$ fluctuations represent episodes of increased mitochondrial potential, i.e., intensified mitochondrial energization. Earlier studies confirmed a clear correlation and linear relationship of $\Delta \Psi_{\mathrm{m}}$ and JC-1 fluorescence only for the red (J-aggregate) emission but not the green (monomer) emission [9, 38]. An even better correlation can, however, be obtained by calculating the ratio of "red" and "green" fluorescence. Therefore, we also plotted the JC-1 ratio (JC-1 red $^{*} 5 / \mathrm{JC}-1_{\text {green}}$; see Fig. 4a).

The $\Delta \Psi_{\mathrm{m}}$ fluctuations typically lasted from several seconds to 1-2 min and the time interval in between two consecutive $\Delta \Psi_{\mathrm{m}}$ surges was highly variable, ranging from a few seconds to several minutes (Fig. $4 \mathrm{a}-\mathrm{c}$ ). $\Delta \Psi_{\mathrm{m}}$ fluctuations were detectable in almost every astrocyte ( $~ 90 \%$ of 124 analyzed), but in a given cell, they occurred only in about $30 \%$ of the mitochondrial population. Mitochondria, not undergoing $\Delta \Psi_{\mathrm{m}}$ fluctuations, remained at a lower level of red JC-1 fluorescence, i.e., lower JC-1 
ratios. The $\Delta \Psi_{\mathrm{m}}$ fluctuations occurred highly synchronized in mitochondrial clusters (Fig. 4a) or neighboring mitochondrial filaments (Fig. 4b) as well as less synchronized in individual mitochondria (Fig. 4c).

Since it has been reported earlier that the red-fluorescing J-aggregate of JC-1 may respond to changes in $\mathrm{pH}$, osmolarity, and $\mathrm{H}_{2} \mathrm{O}_{2}$ levels [15], we confirmed the existence of spontaneous $\Delta \Psi_{\mathrm{m}}$ fluctuations by using the well-established (non-ratiometric) $\Delta \Psi_{\mathrm{m}}$ marker Rh123. As expected, Rh123-labeled mitochondria also showed prominent $\Delta \Psi_{\mathrm{m}}$ fluctuations (Fig. 4d), whose frequencies were, however, two to three times higher than those observed with JC-1 labeling.

The unequivocal identification of those mitochondria undergoing synchronized $\Delta \Psi_{\mathrm{m}}$ fluctuations became possible by so-called activity correlation imaging (ACI, for details, see [24]) performed offline in 40-50 successively taken image frames. Based on a region of interest (individual mitochondrion), only those structures were identified showing a correlation coefficient $>0.5$. Performed on the subcellular level, ACI confirmed that neighboring but also spatially separated mitochondria did undergo highly synchronized $\Delta \Psi_{\mathrm{m}}$ fluctuations (Fig. 5). Also, ACI analysis identified different groups of mitochondria within a single cell which generated synchronized $\Delta \Psi_{\mathrm{m}}$ fluctuations, each at differing frequencies (Fig. 5).

Do cytosolic $\mathrm{Ca}^{2+}$ signals trigger $\Delta \Psi_{\mathrm{m}}$ fluctuations?

Since the synchronized $\Delta \Psi_{\mathrm{m}}$ fluctuations suggest a cytosolic trigger event and intracellular $\mathrm{Ca}^{2+}$ levels $\left(\left[\mathrm{Ca}^{2+}\right]_{\mathrm{i}}\right)$ do modulate mitochondrial metabolism and $\Delta \Psi_{\mathrm{m}}[27,34]$, we tested for a putative effect of $\mathrm{Ca}^{2+}$ influx and $\mathrm{Ca}^{2+}$ release from intracellular stores. Monitoring changes in $\left[\mathrm{Ca}^{2+}\right]_{\mathrm{i}}$ in astrocytes using fluo-3 AM (5 $\mu \mathrm{M}, 30$-min bulk loading) revealed spatiotemporally localized cytosolic $\mathrm{Ca}^{2+}$ transients occurring spontaneously at different sites within the cells (Fig. 6a). These events typically lasted 5-15 s, fluo-3 fluorescence increased up to four-fold (Fig. 6b), and under control conditions, the $\mathrm{Ca}^{2+}$ transients continued to be generated for up to $30 \mathrm{~min}$.

The localized $\mathrm{Ca}^{2+}$ transients persisted in $\mathrm{Ca}^{2+}$-free solutions, suggesting an intracellular origin (Fig. 6c). To verify the involvement of intracellular $\mathrm{Ca}^{2+}$ stores, $\mathrm{Ca}^{2+}$ release from the ER was blocked. Administration of dantrolene $(20-40 \mu \mathrm{M})$ in $\mathrm{Ca}^{2+}$-free solutions antagonized the localized $\mathrm{Ca}^{2+}$ transients in 9 out of 13 astrocytes, and they were abolished in the presence of 2-APB $(100 \mu \mathrm{M}, n=$ 9; Fig. 6d); this confirms that spatially confined $\mathrm{Ca}^{2+}$ release from the ER is the underlying mechanism. The solvent DMSO itself did not affect $\left[\mathrm{Ca}^{2+}\right]_{\mathrm{i}}$ or the localized $\mathrm{Ca}^{2+}$ transients ( $n=4$, Fig. $\left.6 \mathrm{~d}\right)$.
To elucidate whether conditions blocking the localized $\mathrm{Ca}^{2+}$ transients would also abolish the $\Delta \Psi_{\mathrm{m}}$ fluctuations, JC-1-labeled astrocytes underwent the corresponding treatment. Under control conditions, the $\Delta \Psi_{\mathrm{m}}$ fluctuations continued for at least $20 \mathrm{~min}$ before they slowed down and finally ceased ( $n=5$, Fig. 7a) - probably due to the repeated illumination and the associated phototoxicity. Withdrawal of extracellular $\mathrm{Ca}^{2+}$ did not affect the $\Delta \Psi_{\mathrm{m}}$ fluctuations ( $n=7$, Fig. 7 b), but they were antagonized by dantrolene or 2-APB in $\mathrm{Ca}^{2+}$-free solutions. Upon dantrolene application $(20-40 \mu \mathrm{M})$, the $\Delta \Psi_{\mathrm{m}}$ fluctuations slowed down or totally ceased in 18 out of 21 astrocytes within typically $\sim 4$ min (Fig. 7c). 2-APB $(100 \mu \mathrm{M})$ abolished the $\Delta \Psi_{\mathrm{m}}$ fluctuations within $5 \mathrm{~min}$ in eight out of nine astrocytes (Fig. 7d) and markedly slowed them down in the remaining cell. In some astrocytes, the $\Delta \Psi_{\mathrm{m}}$ fluctuations partially recovered upon drug washout. DMSO alone did not affect the $\Delta \Psi_{\mathrm{m}}$ fluctuations $(n=3)$.

Responses to metabolic challenge, excitotoxic stimuli, and metabolic compromise

In the next set of experiments, we asked whether challenging mitochondria modulates the $\Delta \Psi_{\mathrm{m}}$ fluctuations, and at the same time, we screened for homogenous $\Delta \Psi_{\mathrm{m}}$ responses, i.e., JC-1 fluorescence changes, of the mitochondrial population. Mitochondria were exposed to high levels of glutamate and pronounced $\mathrm{Ca}^{2+}$ load to mimic massive neuronal activity/excitotoxicity or to $\mathrm{CN}^{-}$, $\mathrm{NaN}_{3}$, or FCCP to generate conditions of chemical anoxia and mitochondrial impairment. Glutamate $(1 \mathrm{mM}, 4$ $7 \mathrm{~min}$ ) noticeably depolarized mitochondria (decreased the JC-1 ratio) in 8 out of 12 cells, and in 5 of these cells blocked the $\Delta \Psi_{\mathrm{m}}$ fluctuations (Fig. 8a). A second and third exposure to glutamate also induced a pronounced mitochondrial depolarization in the remaining four cells and markedly depressed the $\Delta \Psi_{\mathrm{m}}$ fluctuations in the other seven cells.

Marked changes in $\left[\mathrm{Ca}^{2+}\right]_{\mathrm{i}}$ of glial cells can also be elicited by low extracellular $\mathrm{K}^{+}$levels, a treatment rendering inward-rectifying $\mathrm{K}^{+}$channels $\mathrm{Ca}^{2+}$ permeable $[10,18,20]$. As a result, glial cells experience a pronounced $\mathrm{Ca}^{2+}$ load independent of a preceding marked membrane depolarization. Exposing cultured hippocampal astrocytes to $0.2 \mathrm{mM} \mathrm{K}^{+}$solutions $(5-8 \mathrm{~min})$ depolarized their mitochondria and the $\Delta \Psi_{\mathrm{m}}$ fluctuations slowed down or ceased ( $n=11$, Fig. 8 b).

Mitochondrial uncoupling by FCCP $(1 \mu \mathrm{M}, 1-3 \mathrm{~min})$ led to massive mitochondrial depolarization and abolished the $\Delta \Psi_{\mathrm{m}}$ fluctuations; washout of FCCP partially restored $\Delta \Psi_{\mathrm{m}}$ as well as its spontaneous fluctuations (Fig. 9a, $n=6$ ). Pharmacological inhibition of mitochondrial electron transport by azide $\left(\mathrm{NaN}_{3} ; 2-4 \mathrm{mM}, 1-\right.$ 
Fig. 5 Subcellular activity correlation imaging (ACI) reveals synchronized $\Delta \Psi_{\mathrm{m}}$ fluctuations. a-d ACI analyses identified those mitochondria undergoing synchronized $\Delta \Psi_{\mathrm{m}}$ fluctuations. The left image of each pair displays the JC-1 labeled mitochondria (CCD camera/DualView imaging, red JC-1 channel) and the right image shows the corresponding correlation map. Those mitochondria showing synchronized $\Delta \Psi_{\mathrm{m}}$ fluctuations (correlation coefficient $>0.5$ ) are plotted in the same color. Up to five different large groups of synchronized mitochondria could be identified in a given cell. Comparison of the displayed cells reveals the individual organization and extent of the different groups of synchronized mitochondria. Scaling is identical for all panels
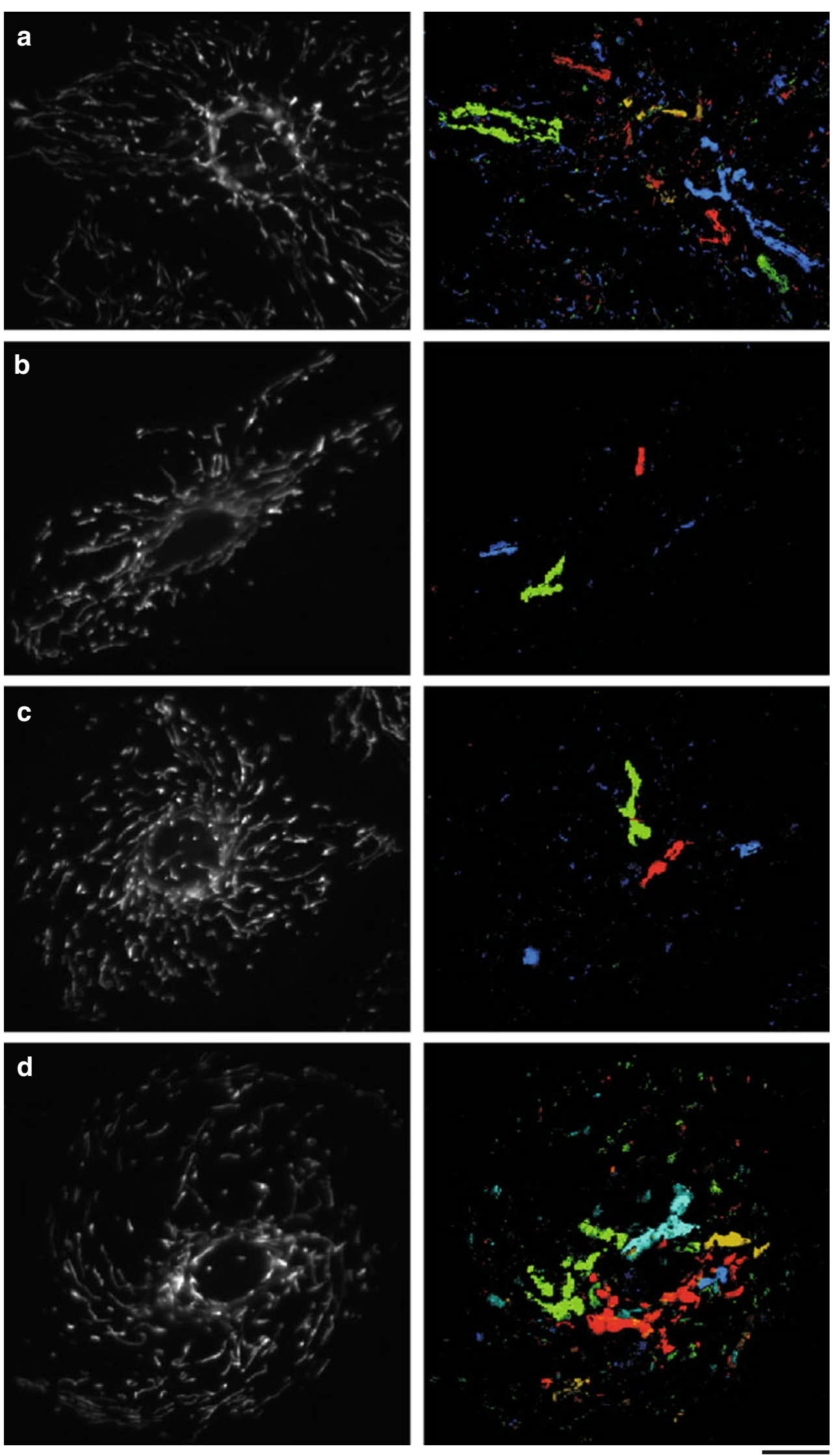

$10 \mu \mathrm{m}$ 
Fig. 6 Fluo-3 recordings confirm the existence of intracellular localized $\mathrm{Ca}^{2+}$ transients. a TPLSM image of a fluo-3-labeled astrocyte and subtraction images (middle and right panel) showing perinuclear $\mathrm{Ca}^{2+}$ transients in pseudo colors. The arrows identify two $\mathrm{Ca}^{2+}$ microdomains; the four regions of interest analyzed are indicated. b Time course of normalized fluo-3 fluorescence analyzed within four perinuclear regions of interest (ROI) of the astrocyte displayed in a. Increases in fluo-3 fluorescence report a rise in $\left[\mathrm{Ca}^{2+}\right]_{\mathrm{i}}$. Time scaling is identical for $\mathbf{b}-\mathbf{d}$. $\mathbf{c}$ Administration of $\mathrm{Ca}^{2+}$-free solutions did not abolish the spatially confined $\mathrm{Ca}^{2+}$ changes, ruling out an influx of $\mathrm{Ca}^{2+}$ from extracellular space as the underlying mechanism. The plotted fluo-3 transients represent $\mathrm{Ca}^{2+}$-microdomains (ROI 1 and 2) recorded by CCD camera/Dual-View imaging from two neighboring astrocytes. d In the presence of 2-APB $(100 \mu \mathrm{M})$, the local $\mathrm{Ca}^{2+}$ fluctuations were reversibly blocked, suggesting an involvement of the ER. The solvent DMSO itself $(0.2 \%)$ did not affect $\left[\mathrm{Ca}^{2+}\right]_{\mathrm{i}}$ a
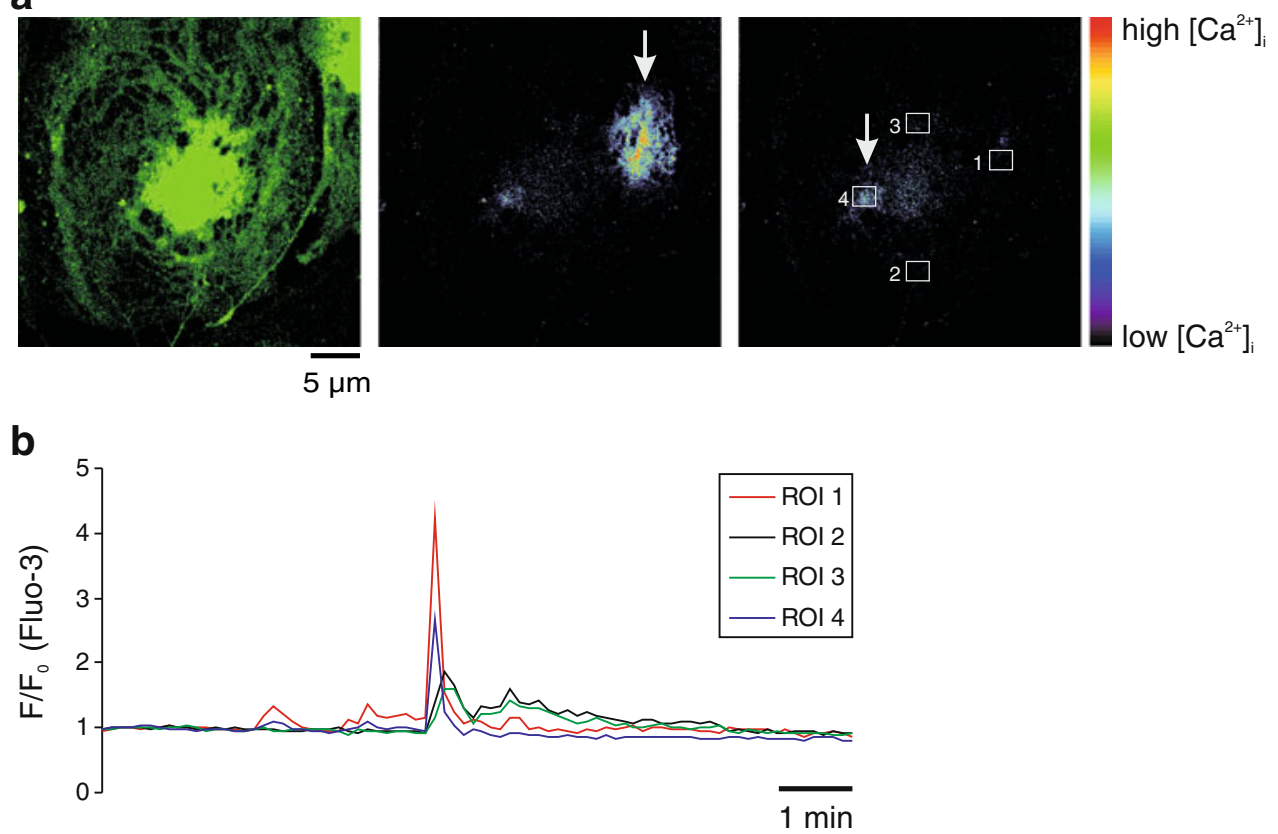

C

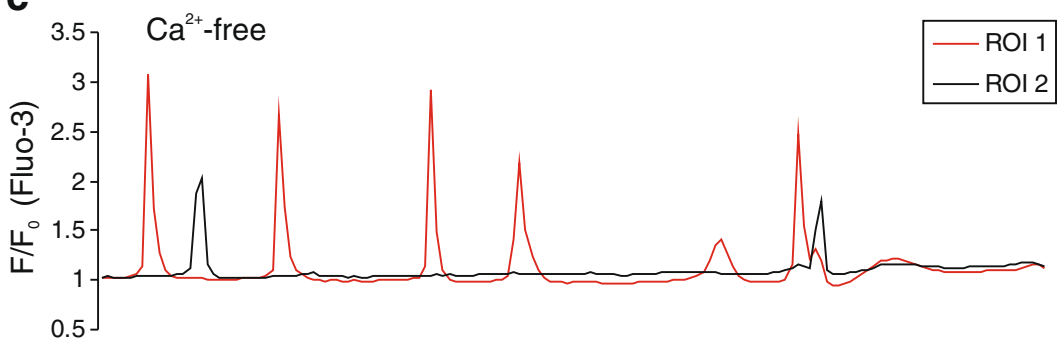

d

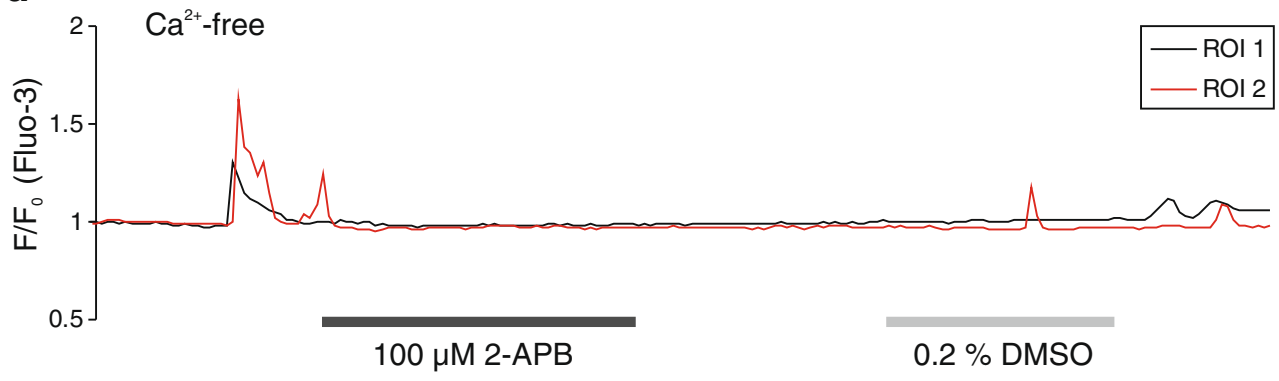

2 min) or cyanide $\left(\mathrm{CN}^{-} ; 1 \mathrm{mM}, 1-2 \mathrm{~min}\right)$ rapidly depolarized mitochondria and abolished the $\Delta \Psi_{\mathrm{m}}$ fluctuations ( $n=13$ and $n=12$, respectively; Fig. 9b, c). The effects of both drugs could be reversed, yet their reversibility decreased with repetitive exposure. Against expectation, upon mitochondrial poisoning with $\mathrm{NaN}_{3}$ or $\mathrm{CN}^{-}$or uncoupling by FCCP not all mitochondria depolarized rapidly; some mitochondria depolarized only slowly and/or remained partly energized. This may suggest heterogeneous time courses of functional impairment within the mitochondrial population of a given cell.
Oxygen withdrawal (95\% $\mathrm{N}_{2}-5 \% \mathrm{CO}_{2}$-aerated solutions for up to $20 \mathrm{~min}$ ) was less efficient. The depolarization of mitochondria was less intense as compared to $\mathrm{NaN}_{3}$ or $\mathrm{CN}^{-}$-mediated poisoning, and only some mitochondria depolarized markedly. Nevertheless, the $\Delta \Psi_{\mathrm{m}}$ fluctuations were consistently abolished or markedly depressed in 11 of 14 cells; upon reoxygenation, the $\Delta \Psi_{\mathrm{m}}$ fluctuations reoccurred (Fig. 9d). The oxygen scavenger Na-sulfite was not added to the solutions, since in initial trials it seemed to promote the formation of JC-1 J-aggregates. 
Fig. $7 \Delta \Psi_{\mathrm{m}}$ fluctuations are triggered by $\mathrm{Ca}^{2+}$-release from the ER. a Under control conditions, $\Delta \Psi_{\mathrm{m}}$ fluctuations were regularly observed. Plotted is the normalized red JC-1 fluorescence as detected by CCDcamera/DualView imaging; time scaling is identical for all panels. b The $\Delta \Psi_{\mathrm{m}}$ fluctuations continued despite withdrawal of extracellular $\mathrm{Ca}^{2+}$, suggesting independence of $\mathrm{Ca}^{2+}$ influx from extracellular space. Solid and dashed lines represent different mitochondria analyzed. c Administration of dantrolene $(20 \mu \mathrm{M})$ antagonized the $\Delta \Psi_{\mathrm{m}}$ fluctuations, confirming an involvement of the ER as intracellular $\mathrm{Ca}^{2+}$ store. d Treatment with 2-APB $(100 \mu \mathrm{M})$ also abolished the $\Delta \Psi_{\mathrm{m}}$ fluctuations of the three mitochondria analyzed

Fig. 8 Challenging mitochondria by voltage-dependent and voltage-independent $\mathrm{Ca}^{2+}$ transients. a Glutamate $(1 \mathrm{mM})$ depolarized the mitochondria and blocked the $\Delta \Psi_{\mathrm{m}}$ fluctuations. Plotted are the normalized red and green JC-1 fluorescence as well as the normalized JC-1 ratio of an individual mitochondrion (CCD camera/Dual-View time-lapse recordings). The TPLSM images show the overlay of red and green JC-1 fluorescence. Scaling is identical for all images/traces. b Lowering $\left[\mathrm{K}^{+}\right]_{\mathrm{o}}$ to $0.2 \mathrm{mM}$ depolarized mitochondria and depressed $\Delta \Psi_{\mathrm{m}}$ fluctuations, indicating that it is not the depolarization of the astrocyte but rather the $\mathrm{Ca}^{2+}$ load which modulates mitochondrial function
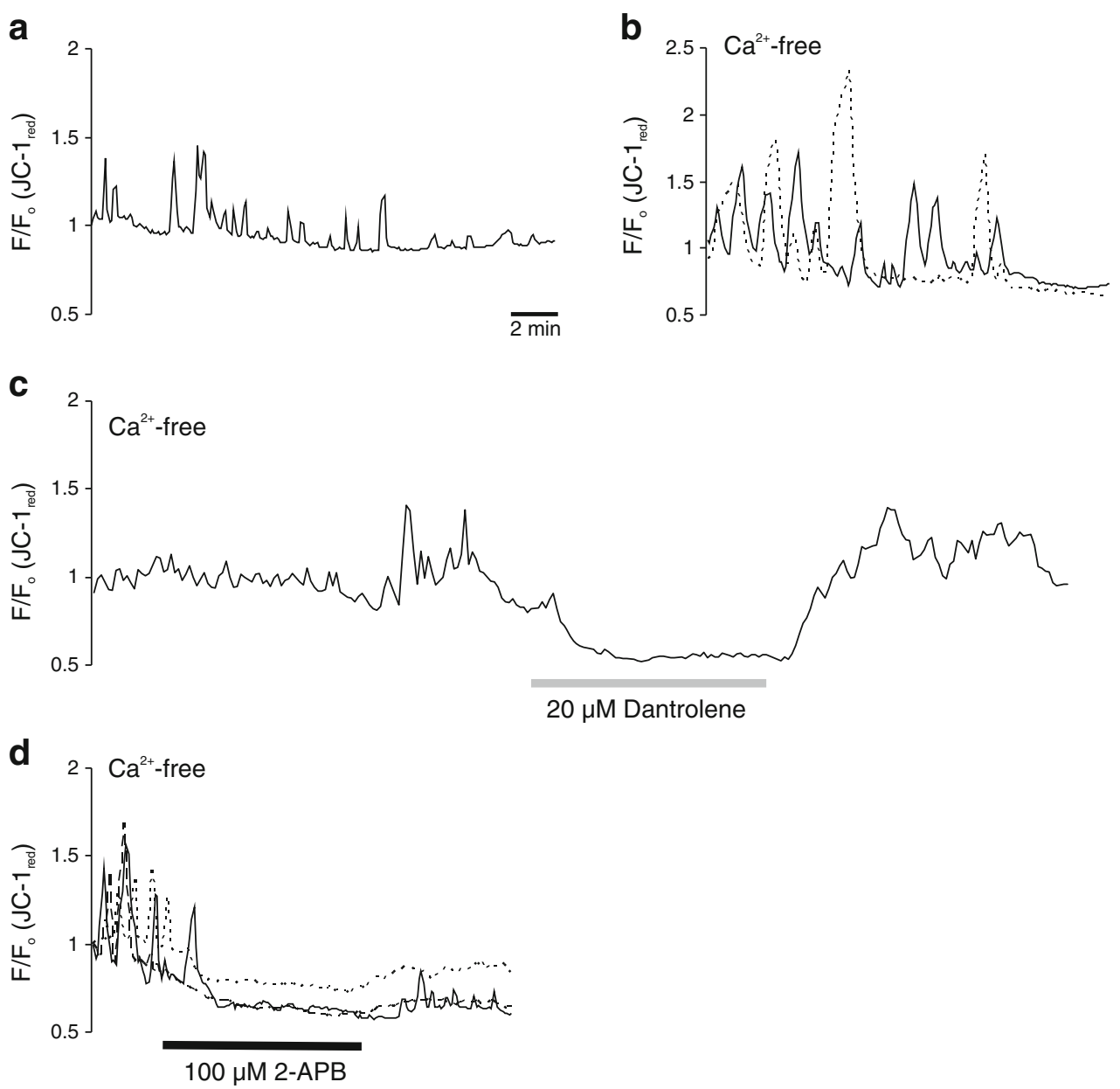

a

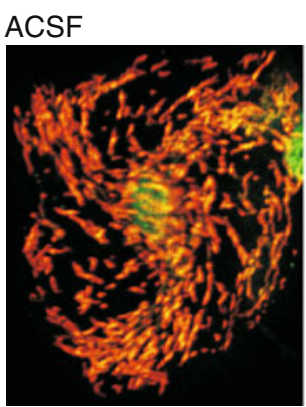

b

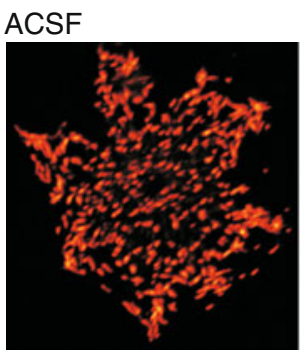

Glutamate

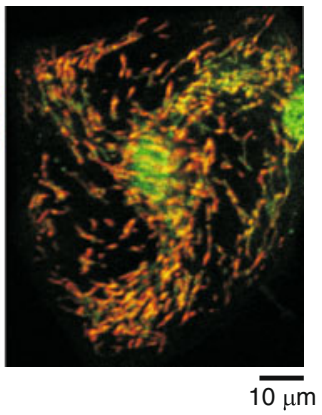

Low $\mathrm{K}^{+}$

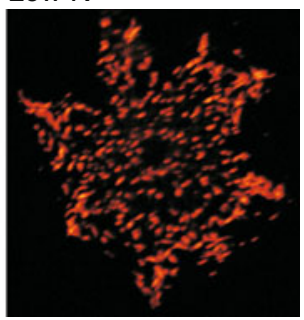

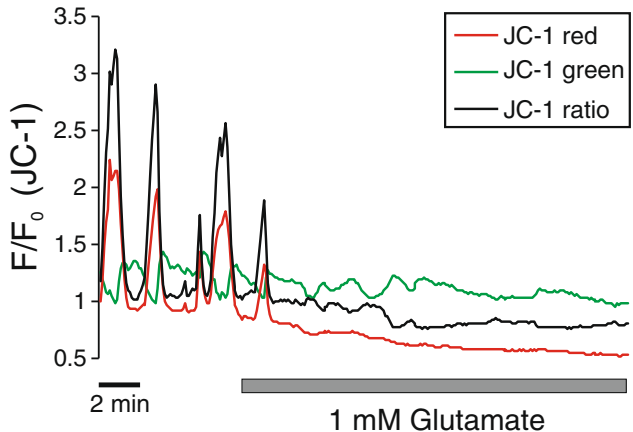

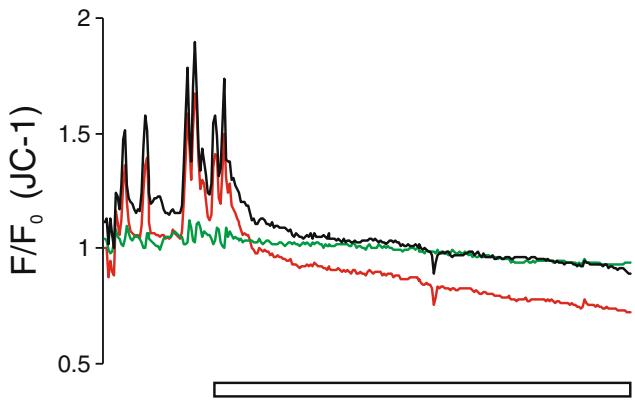

$0.2 \mathrm{mM} \mathrm{K}^{+}$ 
Fig. 9 Pharmacological targeting of mitochondria antagonizes $\Delta \Psi_{\mathrm{m}}$ fluctuations, but does not evoke equally severe $\Delta \Psi_{\mathrm{m}}$ changes within the mitochondrial population. a Mitochondrial uncoupling with FCCP causes a massive loss of $\Delta \Psi_{\mathrm{m}}$, but not the entire mitochondrial population was fully depolarized. TPLSM images show the overlay of red and green JC-1 fluorescence, plotted traces of JC-1 fluorescence were extracted from CCDcamera/Dual-View time-lapse recordings. b Block of complex IV by $\mathrm{NaN}_{3}(2 \mathrm{mM})$ also markedly depolarized mitochondria and the $\Delta \Psi_{\mathrm{m}}$ fluctuations ceased. Some

mitochondria remained, however, partly depolarized. c Inhibiting complex IV by $\mathrm{CN}^{-}(1 \mathrm{mM})$ depolarized mitochondria and blocked the $\Delta \Psi_{\mathrm{m}}$ fluctuations. Again, not all mitochondria showed equally severe depolarizations. d Oxygen withdrawal resulted in a marked depolarization of some mitochondria only. Nevertheless, the $\Delta \Psi_{\mathrm{m}}$ fluctuations were abolished and returned upon reoxygenation
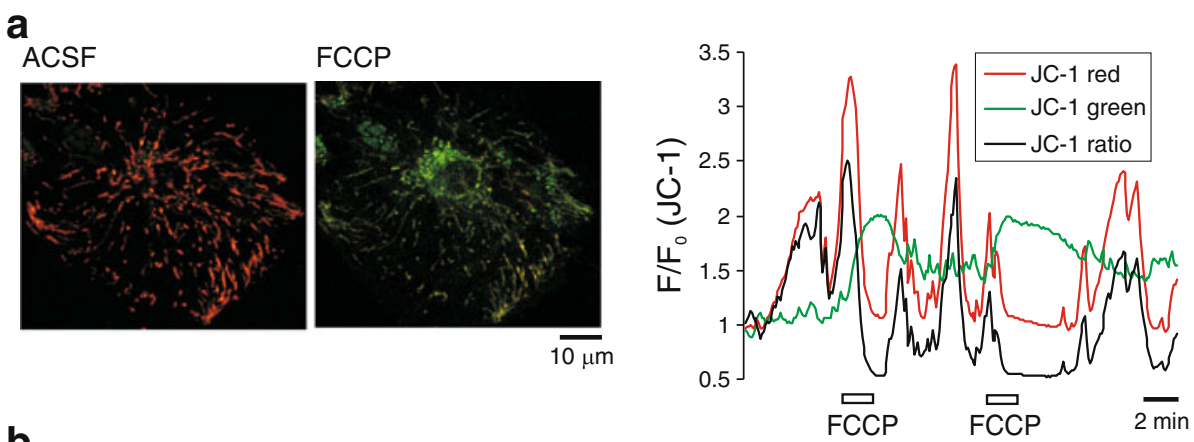

b

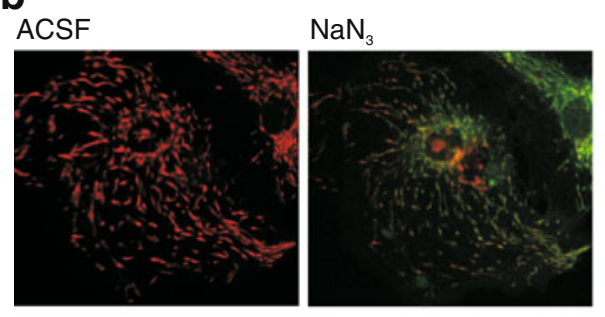

C

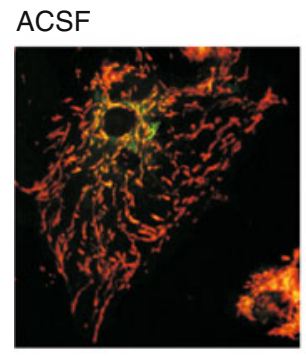

$\mathrm{NaCN}$
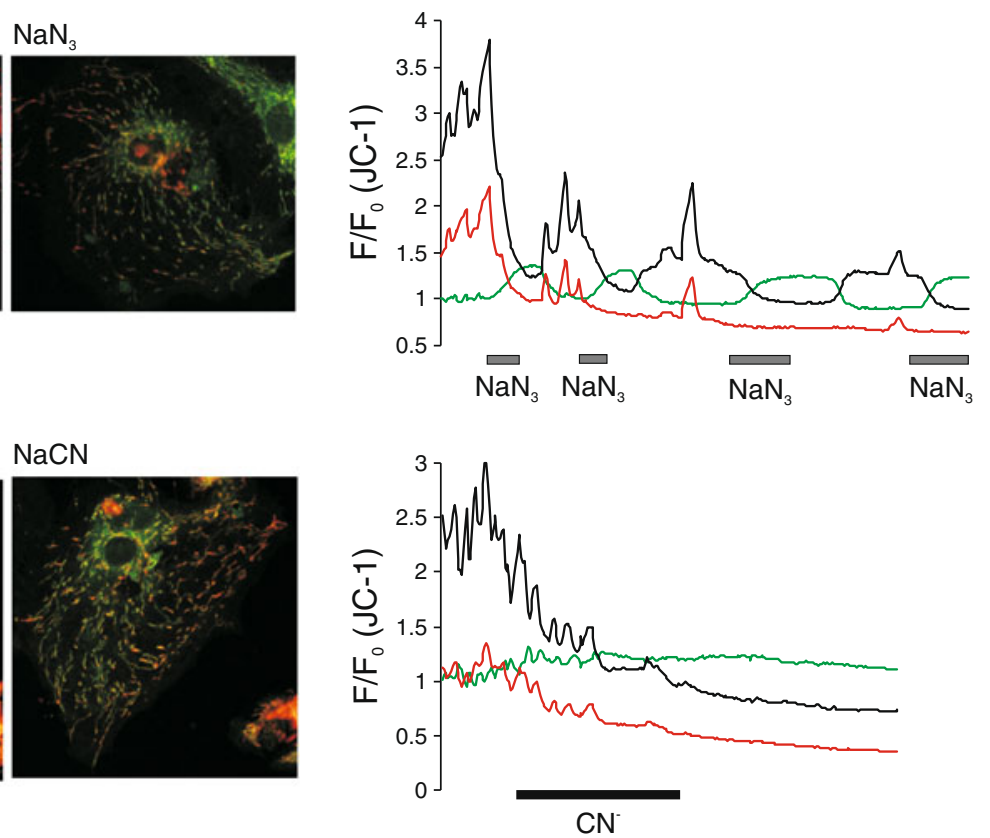

d
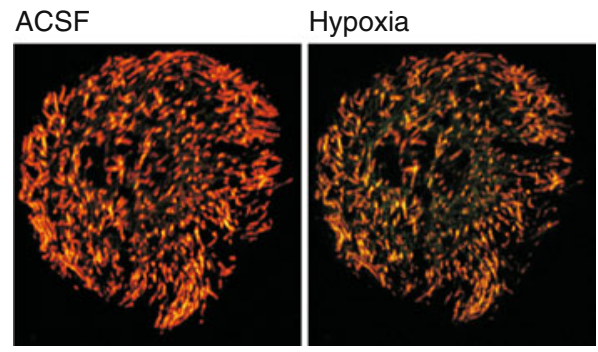

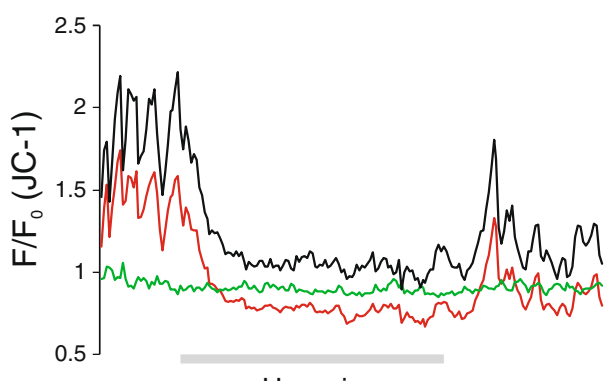

Hypoxia

\section{Discussion}

Our present work confirms the suitability of JC-1 for analyses of the subcellular heterogeneity of mitochondria. Taking advantage of the unique property of JC-1 to spectrally differentiate among functional and depolarized mitochondria [38], we performed ratiometric analyses of red/green JC-1 fluorescence and detected static and dynamic differences in the $\Delta \Psi_{\mathrm{m}}$ of mitochondria in hippocampal astrocytes down to the level of the individual organelle. Possible correlations of mitochondrial size, subcellular loca- tion, and $\Delta \Psi_{\mathrm{m}}$ were analyzed, as were the mitochondrial responses to metabolic challenge and interactions with other cellular organelles. To our knowledge, there is only one prior study on the use of JC-1 in two-photon microscopy [11], which, however, did not include ratiometric analyses.

\section{Suitability of JC-1}

We found JC-1 to label reliably mitochondria and we obtained corresponding staining patterns as with the other mitochondrial markers tested (MitoTracker Red, Rh123). 
Inhomogeneous staining of individual mitochondria due to localized J-aggregates as reported earlier [13, 26] was observed only rarely and does not seem to limit the suitability of JC-1 in hippocampal glial astrocytes. Some limitations may apply, if time courses/kinetics of fast $\Delta \Psi_{\mathrm{m}}$ changes are analyzed, as this may be hampered by the response kinetics of JC-1 (J-aggregate formation/breakdown) which seem to be slower than those of the nonaggregating markers such as Rh123 or tetramethylrhodamine methyl ester (TMRM). Compared with Rh123, we found JC-1 to be less sensitive to photobleaching (see e.g., Fig. 4c, d). This is in line with earlier studies reporting that especially Rh123 is highly susceptible to photobleaching and exhibits strong photo-induced toxicity [6, 31]. In contrast, JC-1 even withstands more than 100 scans performed during either confocal one-photon excitation microscopy or TPLSM imaging [11]. Furthermore, Rh123 concentrations $>1 \mu \mathrm{M}(0.36 \mu \mathrm{g} / \mathrm{ml})$ may interfere with mitochondrial respiration and ATPase activity and concentrations of $>10 \mu \mathrm{M}$ induce mitochondrial swelling [16]. In the case of JC-1, concentrations of up to $550 \mathrm{nM}(0.37 \mu \mathrm{g} / \mathrm{ml})$ have been confirmed not to affect mitochondrial respiration [32]; flow cytometry cell viability assays using even higher concentrations of JC-1 $(10 \mu \mathrm{g} / \mathrm{ml})$ always yielded viability counts of $>95 \%$ [8]. In our experiments, a concentration of $1 \mu \mathrm{g} / \mathrm{ml} \mathrm{JC}-1$ was applied and we did not observe any adverse effects on cell viability or cell shape.

Heterogeneity of mitochondrial shape and $\Delta \Psi_{\mathrm{m}}$

Even though glial cells are less compartmentalized than neurons, their mitochondria are quite heterogeneous in terms of shape, $\Delta \Psi_{\mathrm{m}}$, and subcellular distribution. Mitochondrial shapes ranged from granular to filamentous, resembling conditions found in neuronal, other glial, myocardial, endothelial, and fibroblast preparations [7, 30, 37]. This structural heterogeneity arises from the ability of mitochondria to undergo fusion and fission [1, 2, 42], but also reflects their respective function. Mitochondrial fusion is considered to protect against accumulation of oxidative damage by enabling the exchange of mitochondrial genes and gene products [5]. Furthermore, long mitochondrial filaments were proposed to act as power-transmitting wires [37] and mediate $\mathrm{Ca}^{2+}$ tunneling [7].

Ratiometric JC-1 imaging nicely revealed that $\Delta \Psi_{\mathrm{m}}$ within the mitochondrial population was quite heterogeneous, which is in line with earlier non-ratiometric studies on glia $[7,12]$. Since $\Delta \Psi_{\mathrm{m}}$ determines the functional status of mitochondria, it is not surprising that their metabolism is heterogeneous as well; for example, heterogeneously distributed $\alpha$-ketoglutarate dehydrogenase was reported for mitochondria of cerebellar and cortical astrocytes [41]. Our ratiometric analyses yielded a bell-shaped distribution of $\Delta \Psi_{\mathrm{m}}$ with the majority of mitochondria exhibiting an intermediate $\Delta \Psi_{\mathrm{m}}$. Within an individual mitochondrial structure $\Delta \Psi_{\mathrm{m}}$ appeared homogenous, suggesting that even long filamentous mitochondria are luminally continuous functional entities [12, 30]. Occasional red JC-1 speckles within individual mitochondria are considered artifacts arising from localized J-aggregate formation [13, 26]. Mitochondrial length and $\Delta \Psi_{\mathrm{m}}$ were not correlated, indicating that mitochondrial activity and $\Delta \Psi_{\mathrm{m}}$ are rather determined by intracellular location and interactions with other organelles. It therefore seems worthwhile to differentiate mitochondria according to their very cellular localization and involvement in organelle crosstalk.

Two-photon imaging revealed mitochondrial clustering around the nucleus - comparable to cortical astrocytes [7]and intense colocalization of mitochondria and ER. This clustering and the tendency of a more uniform $\Delta \Psi_{\mathrm{m}}$ among perinuclear mitochondria may serve the high ATP demand of gene expression and subsequent synthesis processes within the ER and Golgi complex. It thus represents an example of local mitochondrial specialization. Preventing a very negative $\Delta \Psi_{\mathrm{m}}$ in perinuclear mitochondria may also serve to limit the formation of reactive oxygen species in that cell region. In addition to the observed $\Delta \Psi_{\mathrm{m}}$ modulation by nuclear proximity and ER interactions, also adjacent acidic vesicles mediate a depolarizing effect on mitochondria [12]. Another example for mitochondrial specialization is that in HeLa cells, HUVEC cells, and hepatocytes, especially peripheral mitochondria, show a proportionally more negative $\Delta \Psi_{\mathrm{m}}$ [7], which may be an adaptation to episodes of cell growth. We observed such a trend in $61 \%$ of astrocytes; yet pooling all data did not confirm a general correlation of the nuclear distance of a mitochondrion and its $\Delta \Psi_{\mathrm{m}}$.

$\Delta \Psi_{\mathrm{m}}$ fluctuations

JC-1 time-lapse recordings indicated clear fluctuations of $\Delta \Psi_{\mathrm{m}}$, during which mitochondria entered a state of higher energization. The time course of the $\Delta \Psi_{\mathrm{m}}$ fluctuations depended on the fluorophore used; they were two to three times faster in Rh123-labeled cells. In accordance, in forebrain neurons, $\Delta \Psi_{\mathrm{m}}$ fluctuations visualized by TMRM show higher frequencies than those recorded with JC-1 [3], which was attributed to different distribution kinetics of both dyes across the inner mitochondrial membrane. Accordingly, JC-1 labeling, due to slow disaggregation of J-aggregates, may underestimate the true kinetics of the $\Delta \Psi_{\mathrm{m}}$ fluctuations. Mechanistically, $\Delta \Psi_{\mathrm{m}}$ fluctuations are on-off transitions of oxidative phosphorylation [3], which also occur in cardiomyocytes and endothelium [14, 22], various neurons [3, 30], human fetal astrocytes [13], and even isolated mitochondria [22, 23, 39, 40]. In our 
experiments, $\sim 30 \%$ of mitochondria produced $\Delta \Psi_{\mathrm{m}}$ fluctuations; in neurons, $55 \%$ of mitochondria were reported to be involved [3]. By applying activity correlation imaging analysis [24] to the subcellular level, we found that neighboring mitochondria, but also groups of mitochondria separated by quite a distance, did undergo synchronized $\Delta \Psi_{\mathrm{m}}$ fluctuations. Also, different groups of synchronized mitochondria could be identified within a given cell.

Though resembling a ubiquitous phenomenon, the detailed mechanisms of the $\Delta \Psi_{\mathrm{m}}$ fluctuations are not entirely understood. They are triggered by increased $\mathrm{Ca}^{2+}$ levels [39] and abolished by $\mathrm{Ca}^{2+}$ chelators [3], suggesting a key role of $\mathrm{Ca}^{2+}$ ions. Indeed, a rise in $\left[\mathrm{Ca}^{2+}\right]_{\mathrm{i}}$ stimulates oxidative phosphorylation [27], and by releasing $\mathrm{Ca}^{2+}$, the ER is capable of modulating mitochondrial function $[19,25$, $28,34]$. Vice versa - as we reported recently -also mitochondria by generating reactive oxygen species are capable of triggering $\mathrm{Ca}^{2+}$ release from the ER [19]. Our present observations strongly suggest that in hippocampal astrocytes, the $\mathrm{Ca}^{2+}$ that drives the $\Delta \Psi_{\mathrm{m}}$ fluctuations is released from the ER rather than entering the cell, which is similar to findings in cardiomyocytes [14]. Increasing $\Delta \Psi_{\mathrm{m}}$ intensifies the electrochemical gradient for $\mathrm{Ca}^{2+}$ uptake and the driving force for ATP synthesis. Accordingly, when astrocytes are exposed to neurotransmitters during neuronal activity, $\mathrm{Ca}^{2+}$ release from intracellular stores and the generation of $\mathrm{Ca}^{2+}$ microdomains might activate and energize nearby mitochondria for the metabolic demand associated with, e.g., $\mathrm{K}^{+}$and neurotransmitter uptake from interstitial space.

\section{Metabolic challenge}

In addition to being part of physiological organelle crosstalk, $\Delta \Psi_{\mathrm{m}}$ fluctuations may also be evoked by pathophysiological events such as excitotoxic stimuli, oxidative stress, and intense illumination/phototoxicity [3, 22, 30], and-at least for non-neuronal mitochondria - transient opening of the permeability transition pore was proposed as another underlying mechanism $[22,23]$. In our experiments, glutamate occasionally triggered $\Delta \Psi_{\mathrm{m}}$ fluctuations in previously "silent" mitochondria. Yet in general, increasing the metabolic load by low $\mathrm{K}^{+}$solutions or glutamate treatment as well as hypoxia, chemical mitochondrial poisoning, or uncoupling blocked the $\Delta \Psi_{\mathrm{m}}$ fluctuations. The antagonistic effect of low $\mathrm{K}^{+}$solutions confirms that a massive $\mathrm{Ca}^{2+}$ load even without a pronounced glial depolarization is sufficient to abolish $\Delta \Psi_{\mathrm{m}}$ fluctuations.

In line with the concept that $\Delta \Psi_{\mathrm{m}}$ fluctuations represent on-off transitions of oxidative phosphorylation, hypoxia, complex IV blockers $\left(\mathrm{CN}^{-}, \mathrm{NaN}_{3}\right)$, and mitochondrial uncoupling (FCCP) abolished the $\Delta \Psi_{\mathrm{m}}$ fluctuations. Such pharmacological inhibition was more efficient than massive $\mathrm{Ca}^{2+}$ influx, obviously due to a direct effect on respiratory complexes and/or the mitochondrial proton gradient. Alto- gether, these results confirm that $\Delta \Psi_{\mathrm{m}}$ fluctuations are restricted to functional mitochondria and hence can be taken as an indication of mitochondrial viability. Nevertheless, it still remains to be elucidated, why only a certain fraction of an intact mitochondrial population produces $\Delta \Psi_{\mathrm{m}}$ fluctuations. For this task, JC-1 seems a valuable tool.

Also, the responses to metabolic compromise varied to some degree within the mitochondrial population. Some mitochondria appeared to remain at a stable relatively polarized potential despite of close proximity to massively depolarizing mitochondria. Clearly, this cannot be explained by differences in $\Delta \Psi_{\mathrm{m}}$ at the time-point of drug administration or uneven intracellular drug distribution. Such heterogeneity was also reported for the timing of the mitochondrial permeability transition in HeLa cells [7]. In part, also the slow disaggregation kinetics of the JC-1 J-aggregates may have contributed to the delayed and/or more subtle responses of some mitochondria to metabolic compromise.

\section{Concluding remarks}

Combining high-resolution imaging with the ratiometric properties of JC-1 revealed that mitochondria in hippocampal astrocytes exhibit a marked heterogeneity that is complemented by structural and functional plasticity. Even though mitochondria are largely autonomous organelles, we found that $\Delta \Psi_{\mathrm{m}}$ is — at least partly-defined and modulated by their immediate cellular environment, which involves interactions with other cellular organelles. Pronounced $\Delta \Psi_{\mathrm{m}}$ fluctuations which seem to indicate mitochondrial viability can clearly arise from spatial proximity to the ER. The localized $\mathrm{Ca}^{2+}$ changes eliciting such $\Delta \Psi_{\mathrm{m}}$ fluctuations were confirmed to represent a possible communication mode in organelle crosstalk. Identification of further cellular events underlying mitochondrial heterogeneity and functional plasticity will be crucial for the understanding of mitochondrial (patho-)physiology in defined cellular compartments. Mitochondrial heterogeneity may give rise to the formation of cytosolic microdomains - such as localized $\mathrm{Ca}^{2+}$ changes, output of reactive oxygen species, and ATP generation - which may critically modulate cellular signaling or even initiate neurodegenerative alterations. In this aspect, the established ratiometric approach of JC-1-based mitochondrial analysis in combination with high-resolution/ two-photon microscopy constitutes a valuable optical tool enabling functional and time-resolved organelle analyses at the level of individual mitochondria and mitochondrial superstructures such as long filaments.

Acknowledgments We thank Belinda Hildebrandt for her excellent technical assistance and Mihai Alevra for providing the algorithm for activity correlated imaging analysis. This study was supported by grants 
of the Deutsche Forschungsgemeinschaft and the BMBF [Cluster of Excellence EXC171, "Microscopy at the Nanometer Range"; DFG Research Center "Center Molecular Physiology of the Brain (CMPB)"].

Open Access This article is distributed under the terms of the Creative Commons Attribution Noncommercial License which permits any noncommercial use, distribution, and reproduction in any medium, provided the original author(s) and source are credited.

\section{References}

1. Bereiter-Hahn J (1990) Behavior of mitochondria in the living cell. Int Rev of Cytology 122:1-63

2. Bossy-Wetzel E, Barsoum MJ, Godzik A, Schwarzenbacher R, Lipton SA (2003) Mitochondrial fission in apoptosis, neurodegeneration and aging. Curr Opin Cell Biol 15:706-716

3. Buckman JF, Reynolds IJ (2001) Spontaneous changes in mitochondrial membrane potential in cultured neurons. J Neurosci 21:5054-5065

4. Cassart D, Fett T, Sarlet M, Baise E, Coignoul F, Desmecht D (2007) Flow cytometric probing of mitochondrial function in equine peripheral blood mononuclear cells. BMC Vet Res 3:25

5. Chan DC (2006) Mitochondria: dynamic organelles in disease, aging, and development. Cell 125:1241-1252

6. Chen LB (1989) Fluorescent labeling of mitochondria. Methods Cell Biol 29:103-123

7. Collins TJ, Berridge MJ, Lipp P, Bootman MD (2002) Mitochondria are morphologically and functionally heterogeneous within cells. EMBO J 21:1616-1627

8. Cossarizza A, Baccarani-Contri M, Kalashnikova G, Franceschi C (1993) A new method for the cytofluorimetric analysis of mitochondrial membrane potential using the $\mathrm{J}$-aggregate forming lipophilic cation 5,5',6,6'-tetrachloro-1,1',3,3'-tetraethylbenzimidazolcarbocyanine iodide (JC-1). Biochem Biophys Res Commun 197:40-45

9. Cossarizza A, Ceccarelli D, Masini A (1996) Functional heterogeneity of an isolated mitochondrial population revealed by cytofluorometric analysis at the single organelle level. Exp Cell Res 222:84-94

10. Dallwig R, Deitmer JW (2002) Cell-type specific calcium responses in acute rat hippocampal slices. J Neurosci Methods $116: 77-87$

11. Dedov VN, Cox GC, Roufogalis BD (2001) Visualisation of mitochondria in living neurons with single- and two-photon fluorescence laser microscopy. Micron 32:653-660

12. Diaz G, Setzu MD, Zucca A, Isola R, Diana A, Murru R, Sogos V, Gremo F (1999) Subcellular heterogeneity of mitochondrial membrane potential: relationship with organelle distribution and intercellular contacts in normal, hypoxic and apoptotic cells. J Cell Sci 112:1077-1084

13. Diaz G, Falchi AM, Gremo F, Isola R, Diana A (2000) Homogeneous longitudinal profiles and synchronous fluctuations of mitochondrial transmembrane potential. FEBS Lett 475:218224

14. Duchen MR, Leyssens A, Crompton M (1998) Transient mitochondrial depolarizations reflect focal sarcoplasmic reticular calcium release in single rat cardiomyocytes. J Cell Biol 142:975-988

15. Duchen MR, Surin A, Jacobson J (2003) Imaging mitochondrial function in intact cells. Methods Enzymol 361:353-389

16. Emaus RK, Grunwald R, Lemasters JJ (1986) Rhodamine 123 as a probe of transmembrane potential in isolated rat-liver mitochondria: spectral and metabolic properties. Biochim Biophys Acta 850:436-448

17. Foster KA, Galeffi F, Gerich FJ, Turner DA, Müller M (2006) Optical and pharmacological tools to investigate the role of mitochondria during oxidative stress and neurodegeneration. Prog Neurobiol 79:136-171

18. Funke F, Dutschmann M, Müller M (2007) Imaging of respiratory-related population activity with single-cell resolution. Am J Physiol Cell Physiol 292:C508-C516

19. Gerich FJ, Funke F, Hildebrandt B, Faßhauer M, Müller M (2009) $\mathrm{H}_{2} \mathrm{O}_{2}$-mediated modulation of cytosolic signaling and organelle function in rat hippocampus. Pflugers Arch 458:937-952

20. Härtel K, Singaravelu K, Kaiser M, Neusch C, Hülsmann S, Deitmer JW (2007) Calcium influx mediated by the inwardly rectifying $\mathrm{K}^{+}$channel Kir4.1 $(\mathrm{KCNJ} 10)$ at low external $\mathrm{K}^{+}$ concentration. Cell Calcium 42:271-280

21. Hepp S, Gerich FJ, Müller M (2005) Sulfhydryl oxidation reduces hippocampal susceptibility to hypoxia-induced spreading depression by activating BK-channels. J Neurophysiol 94:1091-1103

22. Hüser J, Blatter LA (1999) Fluctuations in mitochondrial membrane potential caused by repetitive gating of the permeability transition pore. Biochem J 343:311-317

23. Ichas F, Jouaville LS, Mazat JP (1997) Mitochondria are excitable organelles capable of generating and conveying electrical and calcium signals. Cell 89:1145-1153

24. Junek S, Chen TW, Alevra M, Schild D (2009) Activity correlation imaging: visualizing function and structure of neuronal populations. Biophys J 96:3801-3809

25. Kann O, Kovács R, Heinemann U (2003) Metabotropic receptormediated $\mathrm{Ca}^{2+}$ signaling elevates mitochondrial $\mathrm{Ca}^{2+}$ and stimulates oxidative metabolism in hippocampal slice cultures. J Neurophysiol 90:613-621

26. Kawasaki M, Inokuma H (1999) Contribution of electrostatic cohesive energy in two-dimensional J-aggregation of cyanine dye. J Phys Chem B 103:1233-1241

27. McCormack JG, Halestrap AP, Denton RM (1990) Role of calcium ions in regulation of mammalian intramitochondrial metabolism. Physiol Rev 70:391-425

28. Mironov SL, Ivannikov MV, Johansson M (2005) $\left[\mathrm{Ca}^{2+}\right]_{\mathrm{i}}$ signaling between mitochondria and endoplasmic reticulum in neurons is regulated by microtubules. From mitochondrial permeability transition pore to $\mathrm{Ca}^{2+}$-induced $\mathrm{Ca}^{2+}$ release. $\mathrm{J}$ Biol Chem 280:715-721

29. Müller M, Schmidt J, Mironov SL, Richter DW (2003) Construction and performance of a custom-built two-photon laser scanning system. J Phys Appl D Phys 36:1747-1757

30. Müller M, Mironov SL, Ivannikov MV, Schmidt J, Richter DW (2005) Mitochondrial organization and motility probed by twophoton microscopy in cultured mouse brainstem neurons. Exp Cell Res 303:114-127

31. Poot M, Zhang YZ, Kramer JA, Wells KS, Jones LJ, Hanzel DK, Lugade AG, Singer VL, Haugland RP (1996) Analysis of mitochondrial morphology and function with novel fixable fluorescent stains. J Histochem Cytochem 44:1363-1372

32. Reers M, Smith TW, Chen LB (1991) J-aggregate formation of a carbocyanine as a quantitative fluorescent indicator of membrane potential. Biochemistry 30:4480-4486

33. Reers M, Smiley ST, Mottola-Hartshorn C, Chen A, Lin M, Chen LB (1995) Mitochondrial membrane potential monitored by JC-1 dye. Methods Enzymol 260:406-417

34. Rutter GA, Rizzuto R (2000) Regulation of mitochondrial metabolism by $\mathrm{ER}^{\mathrm{Ca}^{2+}}$ release: an intimate connection. Trends Biochem Sci 25:215-221

35. Salvioli S, Ardizzoni A, Franceschi C, Cossarizza A (1997) JC-1, but not DiOC6(3) or rhodamine 123, is a reliable fluorescent probe to assess $\Delta \Psi$ changes in intact cells: implications for studies on mitochondrial functionality during apoptosis. FEBS Lett 411:77-82

36. Sick TJ, Perez-Pinzon MA (1999) Optical methods for probing mitochondrial function in brain slices. Methods 18:104-108 
37. Skulachev VP (2001) Mitochondrial filaments and clusters as intracellular power-transmitting cables. Trends Biochem Sci 26:23-29

38. Smiley ST, Reers M, Mottola-Hartshorn C, Lin M, Chen A, Smith TW, Steele GD Jr, Chen LB (1991) Intracellular heterogeneity in mitochondrial membrane potentials revealed by a J-aggregateforming lipophilic cation JC-1. Proc Natl Acad Sci U S A 88:3671-3675

39. Vergun O, Reynolds IJ (2004) Fluctuations in mitochondrial membrane potential in single isolated brain mitochondria: modulation by adenine nucleotides and $\mathrm{Ca}^{2+}$. Biophys $\mathrm{J} 87: 3585-3593$
40. Vergun O, Votyakova TV, Reynolds IJ (2003) Spontaneous changes in mitochondrial membrane potential in single isolated brain mitochondria. Biophys J 85:3358-3366

41. Waagepetersen HS, Hansen GH, Fenger K, Lindsay JG, Gibson G, Schousboe A (2006) Cellular mitochondrial heterogeneity in cultured astrocytes as demonstrated by immunogold labeling of alpha-ketoglutarate dehydrogenase. Glia 53:225-231

42. Yaffe MP (2003) The cutting edge of mitochondrial fusion. Nat Cell Biol 5:497-499 